\title{
Minéralogie de l'ancienne mine plomb-zinc de Rabotrath (Lontzen, Belgique)
}

Manuscrit reçu le 4 mai 2016 et accepté le 25 mai 2016

Michel BlONDIEAU ${ }^{1}$, Stéphane PUCCIO ${ }^{2}$, Philippe COMPÈRE $^{3}$ et Frédéric HATERT ${ }^{4}$

${ }^{1}$ Val des Cloches 131, B-6927 Tellin, Belgique

${ }^{2}$ Rue des Fontaines 156, B-4041 Vottem, Belgique

${ }^{3}$ Université de Liège, Laboratoire de Morphologie fonctionnelle et évolutive B6b, B-4000

Liège, Belgique

${ }^{4}$ Université de Liège, Laboratoire de Minéralogie B18, B-4000 Liège, Belgique

\section{Résumé}

Le petit site minier de Rabotrath appartient au district $\mathrm{Pb}-\mathrm{Zn}$ de l'est de la Belgique et se trouve à quelques kilomètres de La Calamine, le plus gros gisement métallique non ferreux belge. La minéralogie de ce gisement n'a jamais été vraiment examinée et fait l'objet de cet article. Outre les espèces minérales classiques associées à cet environnement, comme par exemple la sphalérite, la galène et la smithsonite, on a également découvert trois espèces nouvelles pour la Belgique : la parascholzite, la zaccagnaite-3R, ainsi qu'un minéral de la série plumbogummite-goyazite. Rabotrath devient également la seconde occurrence belge pour deux autres espèces : la chalcophanite et la plumbojarosite. Ces nouvelles observations, qui ont permis le recensement de 25 espèces minérales, viennent ainsi compléter l'inventaire minéralogique du district minier de Moresnet.

Mots clés : gisement $\mathrm{Pb}-\mathrm{Zn}$, Rabotrath, Moresnet, Belgique

\begin{abstract}
The old Rabotrath mine is located close to La Calamine, and belongs to the famous $\mathrm{Pb}-\mathrm{Zn}$ mining district of East Belgium. In this paper, we present a mineralogical description of new samples collected on mine dumps. Besides classical $\mathrm{Pb}-\mathrm{Zn}$ minerals as sphalerite, smithsonite, and galena, we discovered three new mineral occurrences for Belgium: parascholzite, zaccagnaite-(3R), and a mineral of the plumbogummite-goyazite series. Chalcophanite and plumbojarosite were also identified; it's the second Belgian occurrence for these mineral species. The total number of minerals found in Rabotrath is 25 ; the present work significantly contributes to a better knowledge of $\mathrm{Pb}-\mathrm{Zn}$ mineralizations in the Moresnet area.
\end{abstract}

Keywords: Pb-Zn mine, Rabotrath, Moresnet, Belgium

\section{Introduction}

Le village de Rabotrath est situé dans la commune de Lontzen, $2 \mathrm{~km}$ à l'est de Welkenraedt et $4 \mathrm{~km}$ au sud de La Calamine (Kelmis). Il se trouve dans la grande région minière de l'Est de la Belgique, où le zinc, et accessoirement le plomb, ont été extraits de nombreuses mines dont la principale est celle de La Calamine. 
Les premiers travaux réalisés sur le petit site minier de Rabotrath [50 40'08" N ; $6^{\circ} 00^{\prime} 57^{\prime}$ 'E], à 400 m environ au nord-est du village, remontent au moins au $\mathrm{XV}^{\mathrm{e}} \mathrm{S}$ et n'ont consisté à cette époque qu'au glanage de la calamine qui affleurait (le terme «calamine » désigne un mélange de minéraux de zinc oxydés, notamment smithsonite, hémimorphite et willémite). Plus tard, au XVIII ${ }^{\mathrm{e}} \mathrm{S}$, plus de 50 petits bures ont été foncés, et de 1816 à 1919, Rabotrath faisait partie de la concession minière de la Vieille-Montagne (Altenberg) et se trouvait à l'époque en territoire prussien. En 1847, cette société reprend le gisement et fonce trois nouveaux puits atteignant chacun une profondeur légèrement inférieure à 30 mètres. Quelques petits amas calaminaires sont abattus à cette époque puis, en 1852, les travaux sont finalement délaissés.

La production de minerais s'est étalée sur plusieurs siècles et le tonnage des minerais extraits $\mathrm{du} \mathrm{XV}^{\mathrm{e}}$ au $\mathrm{XVIII}{ }^{\mathrm{e}} \mathrm{S}$ est peu connu. Toutefois, sur base de quelques documents d'archive, Dejonghe et al. (1993) signalent une production de 3.500 tonnes de calamine au XVIII ${ }^{\mathrm{e}} \mathrm{S}$. Dans l'acte de renonciation déposé par la société «Union Minière » en 2002, on mentionne une production d'environ 1.000 tonnes de calamine et de quelques dizaines de tonnes de sulfures durant les travaux du XIX ${ }^{\mathrm{e}} \mathrm{S}$. La production totale de Rabotrath serait donc de l'ordre de 5.000 tonnes de calamine, ce qui est évidemment fort peu en comparaison avec le gros gisement de La Calamine qui aurait, à lui seul, fourni 1,9 millions de tonnes de minerai d'où furent extraites 760.000 tonnes de zinc métal (Dejonghe et al., 1993).

Une lithographie d'Adolphe Maugendre (1809-1895), réalisée pour le compte de «la Société anonyme des Mines et fonderies de zinc de la Vieille Montagne (1850-1851) » et imprimée à Paris par Auguste Bry, montre le site industriel tel qu'il était au XIX ${ }^{\mathrm{e}} \mathrm{S}$ (Fig. 1). Maugendre était connu pour sa minutie du détail; il est encore possible d'observer, aujourd'hui, le bâtiment principal (à gauche sur la lithographie; Fig. 2) ainsi que le chemin creux qui le longe et qui se dirige vers Poppelsberg, un autre site minier. De même, de l'autre côté de la route, les petites mares le long du «lontzenerbach », sont ce qu'il reste des trois bassins de lavage du minerai, bien visibles à l'avant-plan de la lithographie. Par contre, tous les autres bâtiments miniers ont disparu, et il ne reste que de maigres déblais localisés autour du puits principal, plus ou moins rebouché et pas encore sécurisé par Union Minière.

Aujourd'hui le site, en milieu rural, est couvert par une prairie et, localement, par une «pelouse calaminaire » aux endroits où le sol est le plus chargé en métaux. Le site est repris dans la liste des Sites de Grand Intérêt Biologique (SGIB) de la région Wallonne ( $\mathrm{N}^{\circ} 2455$ ), car on y observe de nombreuses plantes métallophytes dont la plus emblématique est très certainement la pensée calaminaire (viola calaminaria Lej), une plante typique des sols riches en zinc.

Récemment, de nouvelles fouilles ont été réalisées non loin de l'ancien puits principal, et ont permis de recueillir des échantillons de minerais dans lesquels ont pu être recensées plus d'une vingtaine d'espèces minérales. Outre la calamine relativement abondante, on observe également une brèche avec éléments de «schalenblende » (= sphalérite rubanée) cimentés par de la sidérite, ce qui correspond bien aux descriptions faites par Dejonghe et al. (1993). 
Cet article a pour but de décrire précisément les associations minérales du gisement $\mathrm{Pb}-\mathrm{Zn}$ de Rabotrath, et d'établir ainsi l'inventaire détaillé des espèces observées.

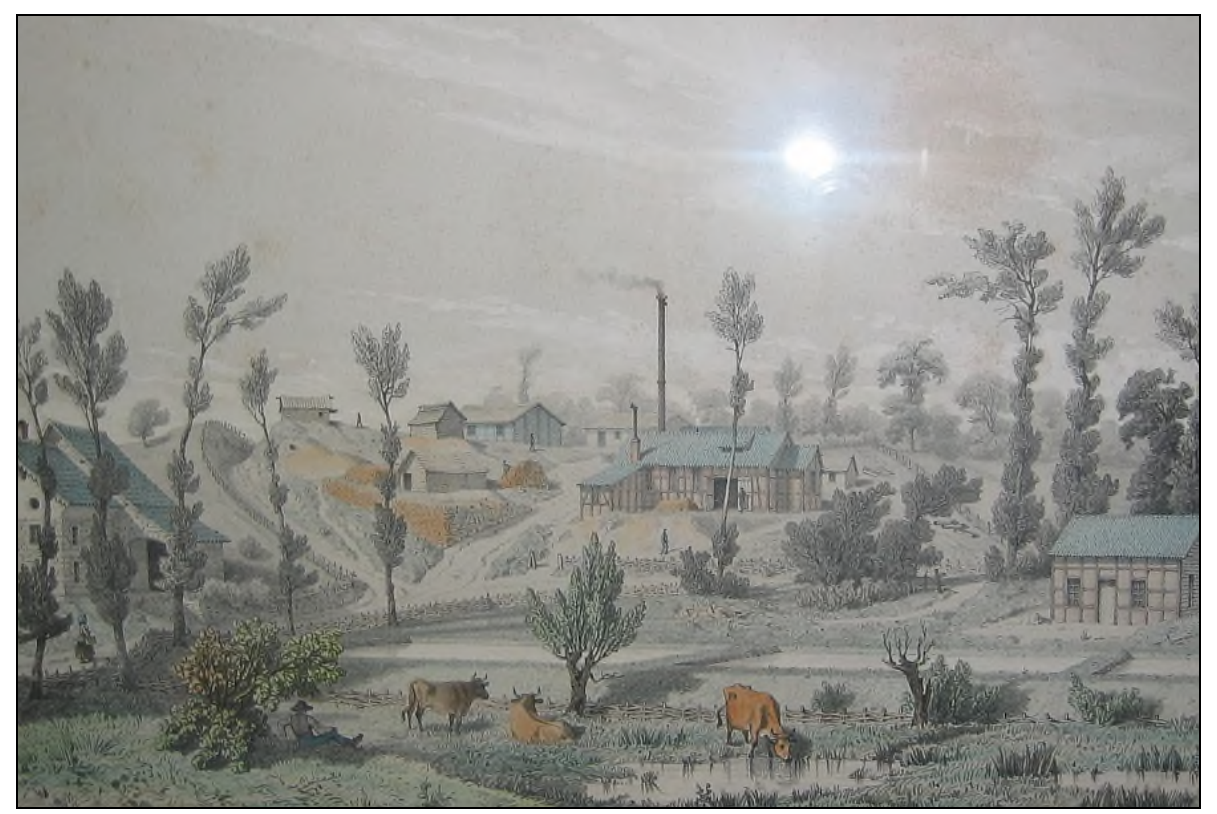

Fig. 1 : Lithographie d'Adolphe Maugendre (1850-1851) illustrant le site minier de Rabotrath (photo: M. Blondieau).

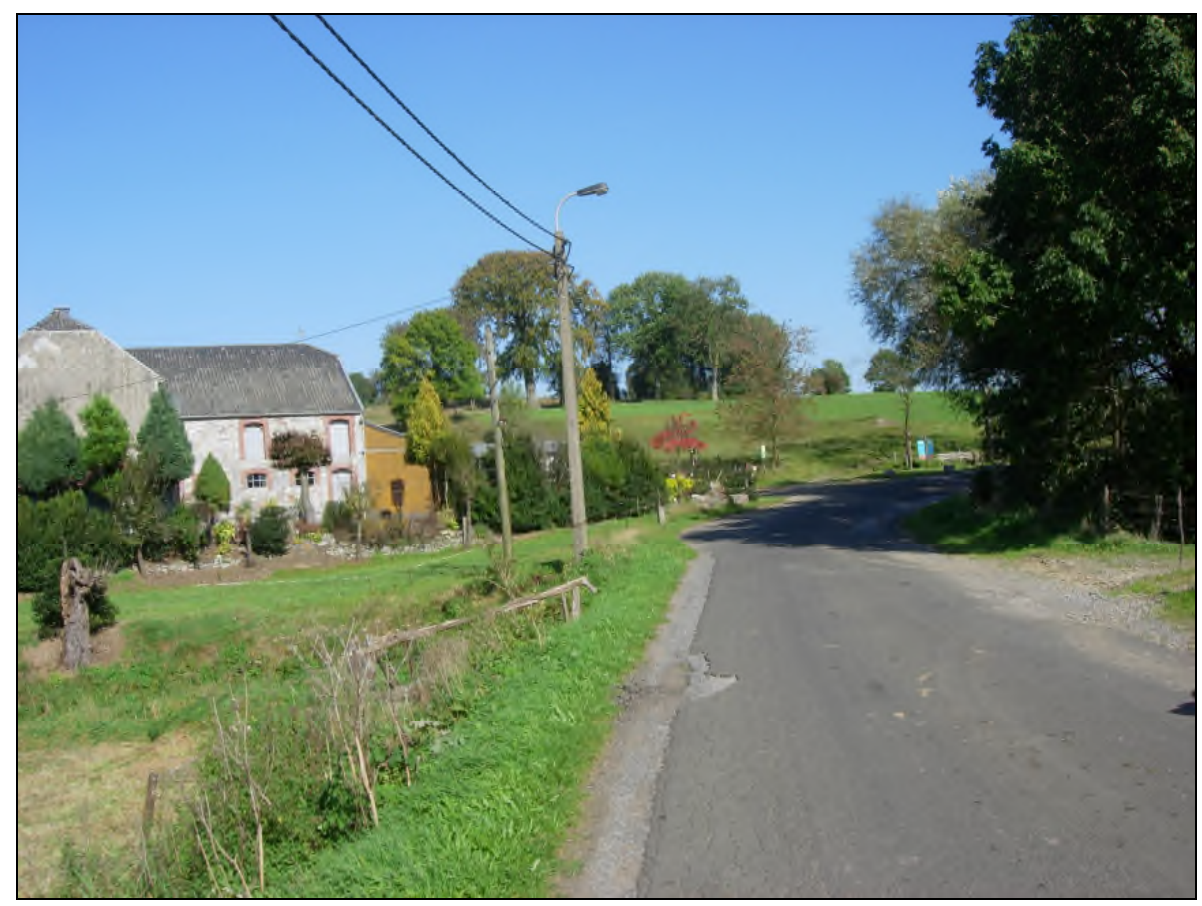

Fig. 2 : Village de Rabotrath. La maison à gauche est l'ancien bâtiment principal de la mine, et l'ancien site minier est visible à l'arrière-plan (photo : M. Blondieau, octobre 2014). 


\section{Contexte géologique}

Les minéralisations décrites font partie d'un district métallifère localisé dans le synclinorium de Verviers. Ce synclinorium constitue le prolongement oriental du synclinorium de Dinant, et est délimité à l'ouest par la ville de Liège, à l'est par Aachen, au sud-est par le Massif de Stavelot, et au nord-ouest par la faille des Aguesses-Asse qui est un tronçon de la faille du Midi aux environs de Liège (Dejonghe, 1998).

Deux phases de dépôt sont observées dans cette région. La première comporte des shales du Dévonien supérieur (Famennien, Fm), des calcaires parfois dolomitisés du Carbonifère inférieur (Tournaisien, Tn; Viséen, V), ainsi que des shales et grès du Carbonifère supérieur (Namurien, Nm ; Fig. 3). Après plissement de ces roches au cours de l'orogenèse varisque, une seconde phase de dépôt plus tardive a permis la formation de roches mésozoïques discordantes et non déformées.

Comme le montre la carte géologique simplifiée de la région de Rabotrath (Fig. 3), les roches paléozoïques ont également été fortement faillées, et l'on peut observer deux directions principales pour ces failles. Les grandes failles, orientées NE-SO, sont liées à des charriages produits par l'orogenèse varisque, alors que les failles de décrochement NNW-SSE sont liées à la formation plus récente du Graben du Rhin, relativement proche de cette région (Dejonghe, 1998). La majorité des minéralisations $\mathrm{Pb}-\mathrm{Zn}$ sont localisées le long des failles NNW-SSE.

À Rabotrath, la minéralisation forme un petit amas le long d'un contact entre d'une part, des calcaires viséens, et d'autre part, des shales et des grès namuriens. Cet amas est localisé sur le passage d'une faille transversale se prolongeant au nord-ouest à Poppelsberg et à Lontzen, où d'autres corps minéralisés ont été exploités (Fig. 3 ; Dejonghe et al., 1993). La minéralisation est composée exclusivement de calamine ferrugineuse qui, en profondeur, fait place à de la sidérite, de la pyrite et de l'argile noire dans laquelle se trouvent dispersés des rognons de sphalérite rubanée («schalenblende ») et de galène. Dejonghe et al. (1993) mentionnent la présence de huit espèces minérales à Rabotrath : «calamine», calcite, cérusite, galène, « limonite », pyrite $s$. $l$. (= pyrite ou marcasite), sidérite et sphalérite. 


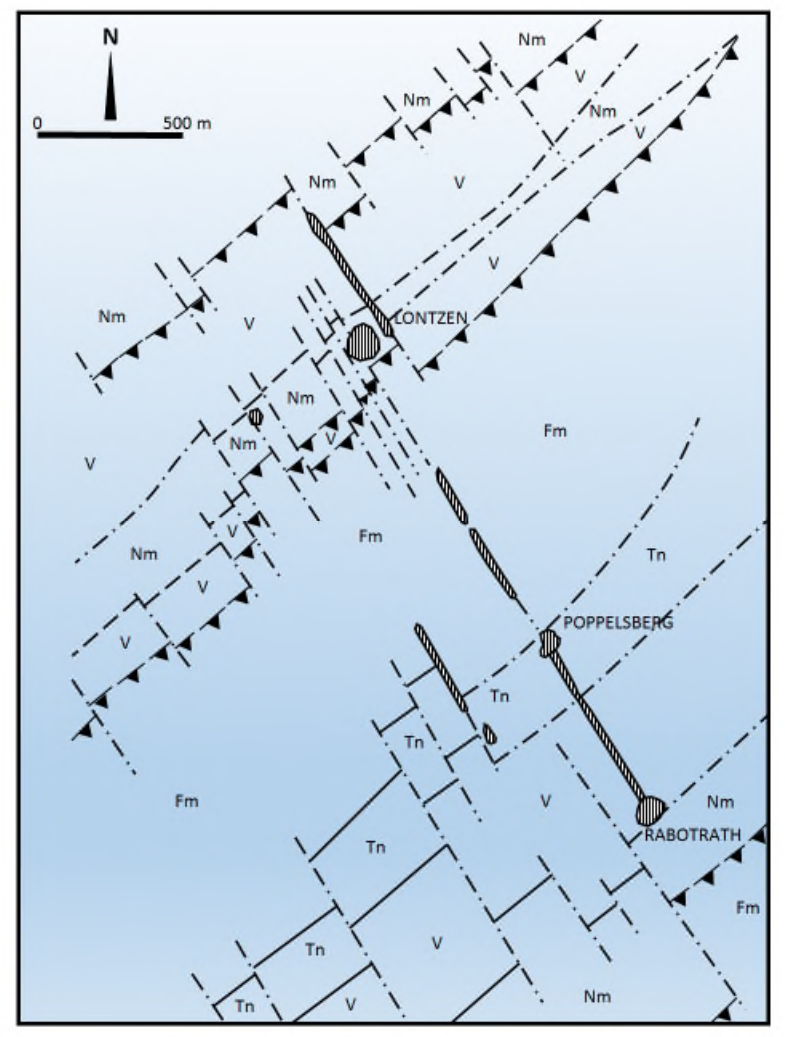

Fig. 3: Carte géologique montrant la localisation des minéralisations de Rabotrath, Poppelsberg et Lontzen (d'après Dejonghe et al., 1993). Fm = Famennien, $\mathrm{Tn}=$ Tournaisien, $\mathrm{V}=$ Viséen, $\mathrm{Nm}=$ Namurien. Les filons et amas métallifères sont représentés en hachurés verticaux.

\section{Descriptions minéralogiques}

Quelques petites fouilles réalisées non loin de l'ancien puits principal nous ont permis de recueillir des échantillons de minerais dans lesquels ont pu être observées plus d'une vingtaine d'espèces minérales. Outre la calamine relativement abondante, on trouve aussi une brèche avec éléments de «schalenblende » cimentés par de la sidérite, ce qui correspond bien aux descriptions faites par Dejonghe et al. (1993).

Les minéraux ont été soumis à une analyse par diffraction des rayons $\mathrm{X}$ sur un diffractomètre de poudres PANALYTICAL PW-3710 utilisant la radiation $\mathrm{FeK}_{\alpha}(\lambda=1,9373 \AA)$. Lorsque les quantités de matière étaient très faibles, un diffractomètre à 4 cercles Agilent Technologies Xcalibur, équipé d'un détecteur EOS et utilisant la radiation $\operatorname{MoK}_{\alpha}(\lambda=0,7107 \AA)$, a été utilisé en mode «Debye-Scherrer ». Les paramètres de maille de la zaccagnaite-3R ont été calculés grâce au programme d'affinement par moindres carrés LCLSQ version 8.4 (Burnham, 1991). Un microscope électronique à balayage environnemental ESEM XL 30 FEI (Cat $\mu$, Centre for Applied Technology in Microscopy, Université de Liège), a été utilisé pour obtenir des images en mode électrons secondaires, ainsi que pour récolter des spectres EDS («Energy Dispersive X-ray Spectrometry») permettant de fournir des analyses chimiques qualitatives ou semi-quantitatives. 


\subsection{Allophane, $\mathrm{Al}_{2} \mathrm{O}_{3}\left(\mathrm{SiO}_{2}\right)_{1,3-2,0} \cdot \mathbf{2 , 5 - 3 , 0 \mathrm { H } _ { 2 } \mathrm { O }}$}

L'allophane forme des enduits mamelonnés incolores à éclat vitreux, ou se présente en masses brunâtres à cassures conchoïdales, d'éclat résineux. Le minéral est fragile et se fragmente facilement; son identification est confirmée par le diffractogramme de poudres de rayons $\mathrm{X}$ qui montre deux pics larges à 3,30 et 2,25 Å. Les spectres EDS réalisés sur les parties incolores et les parties crème sont tout à fait comparables et mettent en évidence l'aluminium, le silicium et l'oxygène.

\subsection{Anglésite, $\mathrm{PbSO}_{4}$}

L'anglésite est visuellement observée en minuscules cristaux prismatiques blancs, dans la brèche à sulfures cimentée par la sidérite. L'examen au microscope électronique à balayage permet de visualiser des petits prismes d'une trentaine de micromètres de longueur (Fig. 4A), développés sur des cristaux de sidérite associés à la marcasite. Ces cristaux montrent les formes $\{011\}$ et $\{100\}$ dominantes, associées aux facettes $\{110\},\{101\}$ et $\{111\}$ moins développées (Fig. 4B). Le spectre EDS indique la présence des éléments $\mathrm{Pb}, \mathrm{S}$ et $\mathrm{O}$, confirmant ainsi l'identification du minéral. L'anglésite a également été identifiée par diffraction des rayons $\mathrm{X}$, en mélange avec la plumbojarosite recouvrant des échantillons de marcasite.

A
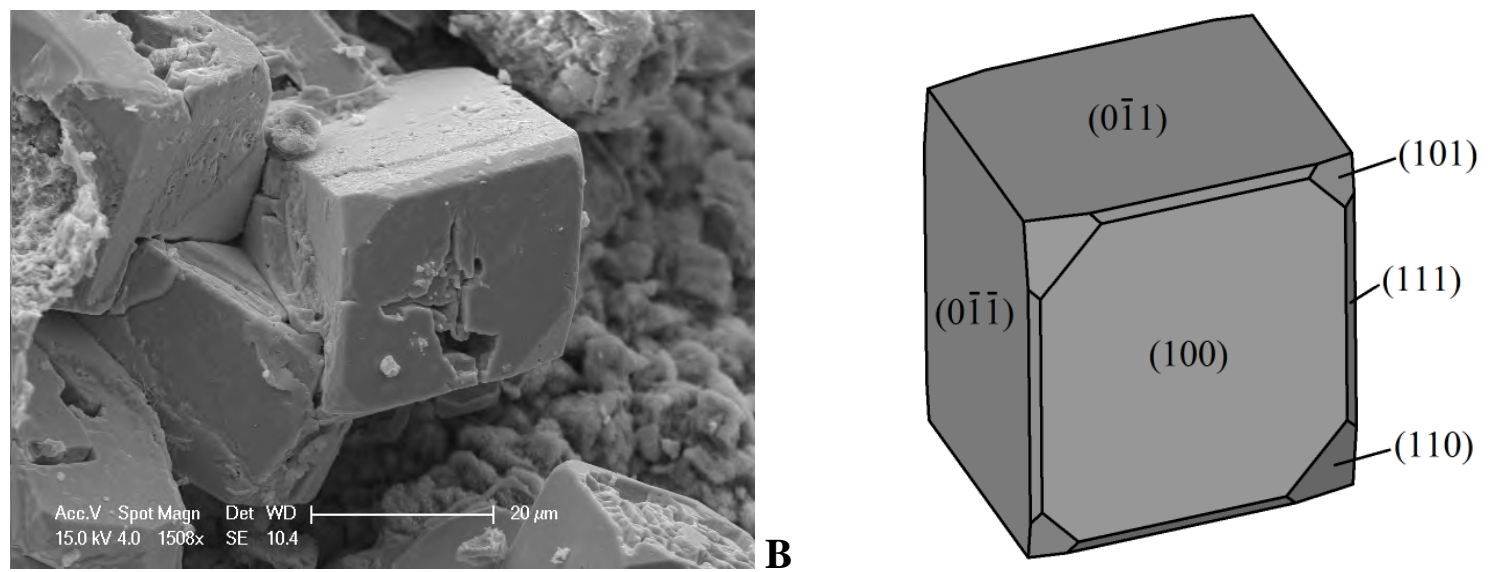

Fig. 4 : Anglésite de Rabotrath (Échantillon RAB-15). A. Photographie au microscope électronique à balayage, mode électrons secondaires. B. Dessin de la morphologie du cristal, caractérisé par la combinaison $\{011\}\{100\}\{110\}\{101\}\{111\}$.

\subsection{Aragonite, $\mathrm{CaCO}_{3}$}

L'aragonite forme de minuscules géodes dans la sidérite constituant le ciment de la brèche à sulfures. Les cristaux sont aciculaires, incolores à blancs, et peuvent atteindre $100 \mu \mathrm{m}$ de longueur (Fig. 5). L'analyse chimique qualitative montre la présence des seuls éléments Ca, C et $\mathrm{O}$, confirmant ainsi l'identification visuelle. 


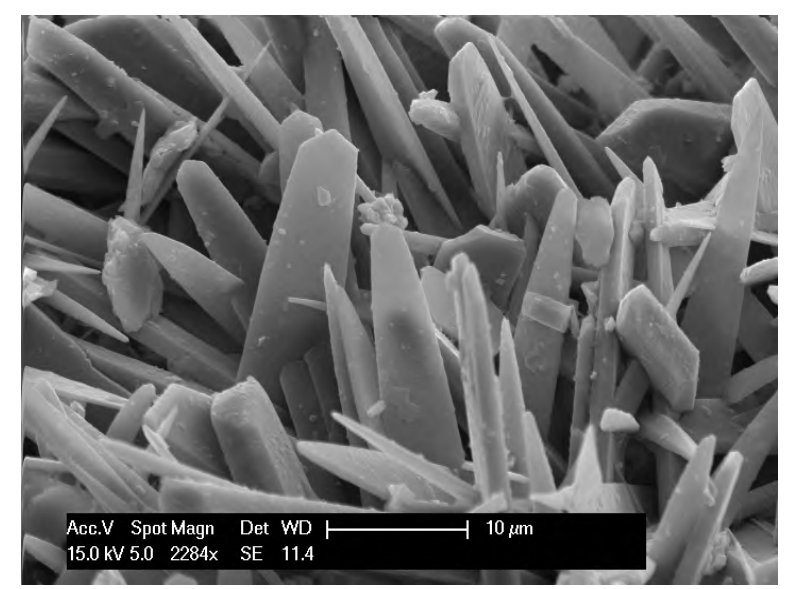

Fig. 5 : Aragonite de Rabotrath (Échantillon RAB-04, photographie au microscope électronique à balayage, mode électrons secondaires).

\subsection{Calcite, $\mathrm{CaCO}_{3}$}

La calcite a été déterminée visuellement, et accompagne parfois la sphalérite rubanée. Le minéral n'a pas été observé en beaux cristaux, et forme généralement des encroûtements blanchâtres sur les sulfures.

\subsection{Cérusite, $\mathrm{PbCO}_{3}$}

La cérusite a été identifiée visuellement en fort petits cristaux grisâtres, déposés sur la galène altérée. Sous le microscope électronique à balayage, ces cristaux sont mal développés et ne dépassent guère les $20 \mu \mathrm{m}$ de diamètre (Fig. 6A). D'autres cristaux incolores à blanc, qui peuvent atteindre plusieurs millimètres de longueur, ont également été identifiés visuellement dans des cavités de calamine. Ces cristaux, déposés sur la smithsonite, montrent un habitus cristallin prismatique terminé par une pyramide, ce qui pourrait suggérer la présence d'une macle pseudo-hexagonale (Fig. 6B). L'analyse chimique qualitative montre la présence des seuls éléments $\mathrm{Pb}, \mathrm{C}$ et $\mathrm{O}$, confirmant ainsi l'identification.
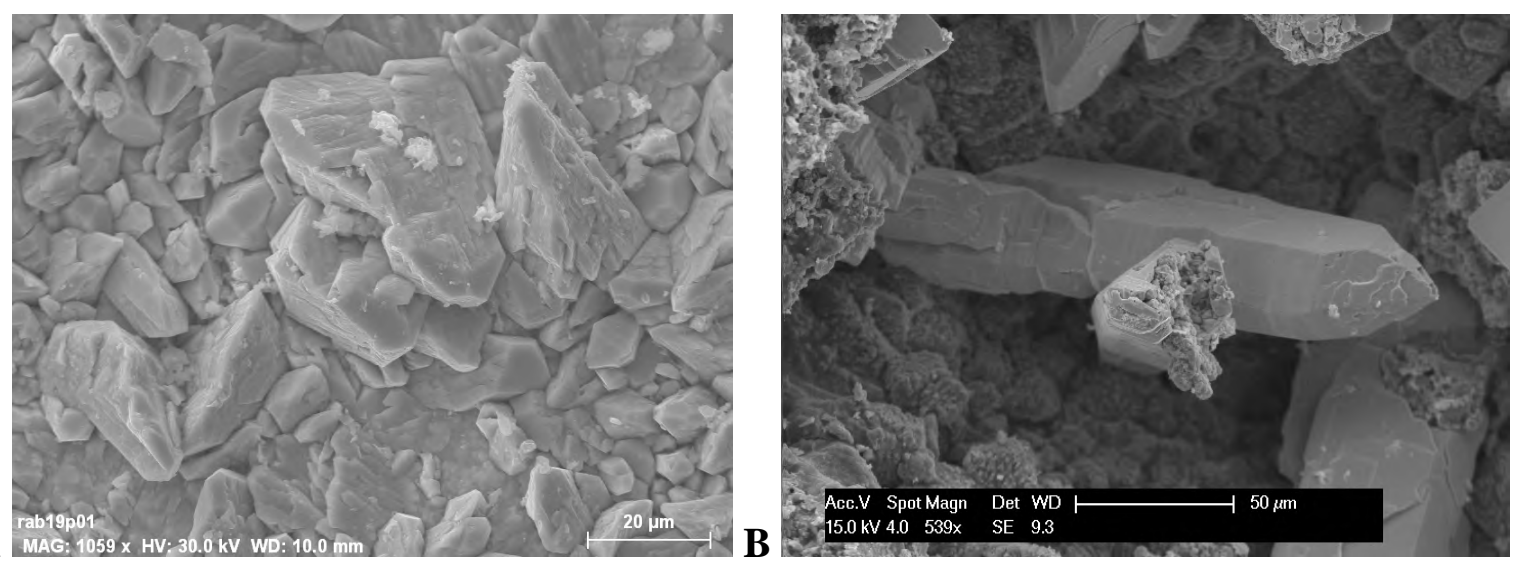

Fig. 6 : Cérusite de Rabotrath (Photographies au microscope électronique à balayage, mode électrons secondaires). A. Échantillon RAB-20 montrant de petits cristaux déposés sur la galène. B. Échantillon RAB-16 caractérisé par de grands prismes développés dans la calamine. 


\subsection{Chalcophanite, $\mathrm{ZnMn}^{4+}{ }_{3} \mathrm{O}_{7} .3 \mathrm{H}_{2} \mathrm{O}$}

La chalcophanite est un minéral brun noir formant des enduits concordants avec les couches de smithsonite. Le minéral apparaît brun et terreux en surface, mais est plus noir et compact en profondeur. Ces encroûtements sont relativement fréquents à Rabotrath ; l'identification a été confirmée par diffraction des rayons $X$, ainsi que par le spectre EDS qui indique la présence de l'oxygène, du manganèse et du zinc.

Des petites boules noires de $100 \mu \mathrm{m}$ de diamètre, agglomérées en petits amas dans les fentes de la goethite brune, ont également été identifiées par diffraction des rayons $\mathrm{X}$ comme chalcophanite. Les images au microscope électronique à balayage montrent qu'elles sont couvertes d'une multitude de cristaux d'aspect lamellaire, empilés en tous sens et formant les petites sphérules (Fig. 7). Ces lamelles ne dépassent pas $10 \mu \mathrm{m}$ de longueur, et leur spectre EDS confirme la présence des éléments $\mathrm{Mn}, \mathrm{O}$ et $\mathrm{Zn}$.

A
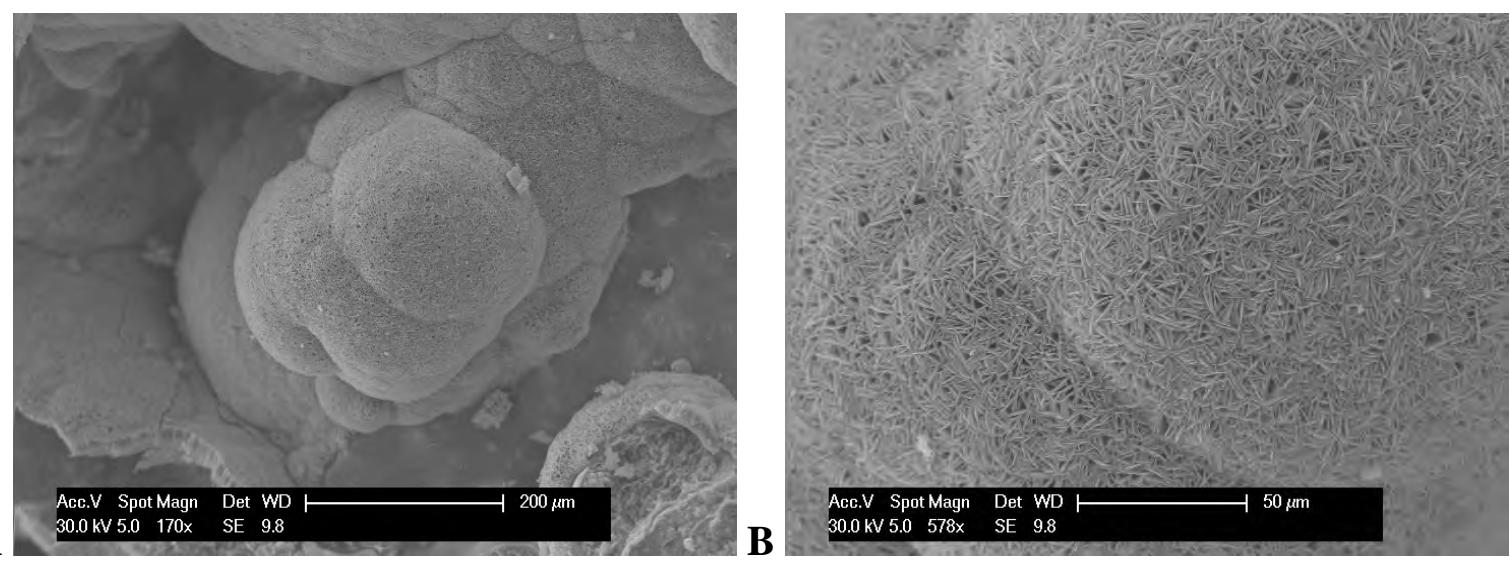

Fig. 7 : Chalcophanite de Rabotrath (Échantillon RAB-17, photographie au microscope électronique à balayage, mode électrons secondaires).

En Belgique, la chalcophanite est un minéral rare, qui n'a été signalée qu'une fois à Beez par Blondieau et al. (2012). Il est cependant possible que ce minéral soit présent dans de nombreux autres gisements, au voisinage de la smithsonite; son aspect terreux le rend certainement peu attrayant.

\subsection{Dundasite, $\mathrm{PbAl}_{2}\left(\mathrm{CO}_{3}\right)_{2}(\mathrm{OH})_{4} \cdot \mathrm{H}_{2} \mathrm{O}$}

La dundasite forme des amas de petites fibres blanches, associées à des plages d'allophane vitreuse incolore et à des plages d'allophane brune résineuse. Sous le microscope électronique à balayage, les fibres se présentent en lattes de un ou $2 \mu \mathrm{m}$ de large et de $30 \mu \mathrm{m}$ de longueur maximale (Fig. 8). L'identification est confirmée par le diffractogramme de poudres de rayons $\mathrm{X}$ et par le spectre EDS qui indique la présence majoritaire des éléments $\mathrm{O}, \mathrm{C}, \mathrm{Al}$ et $\mathrm{Pb}$. 


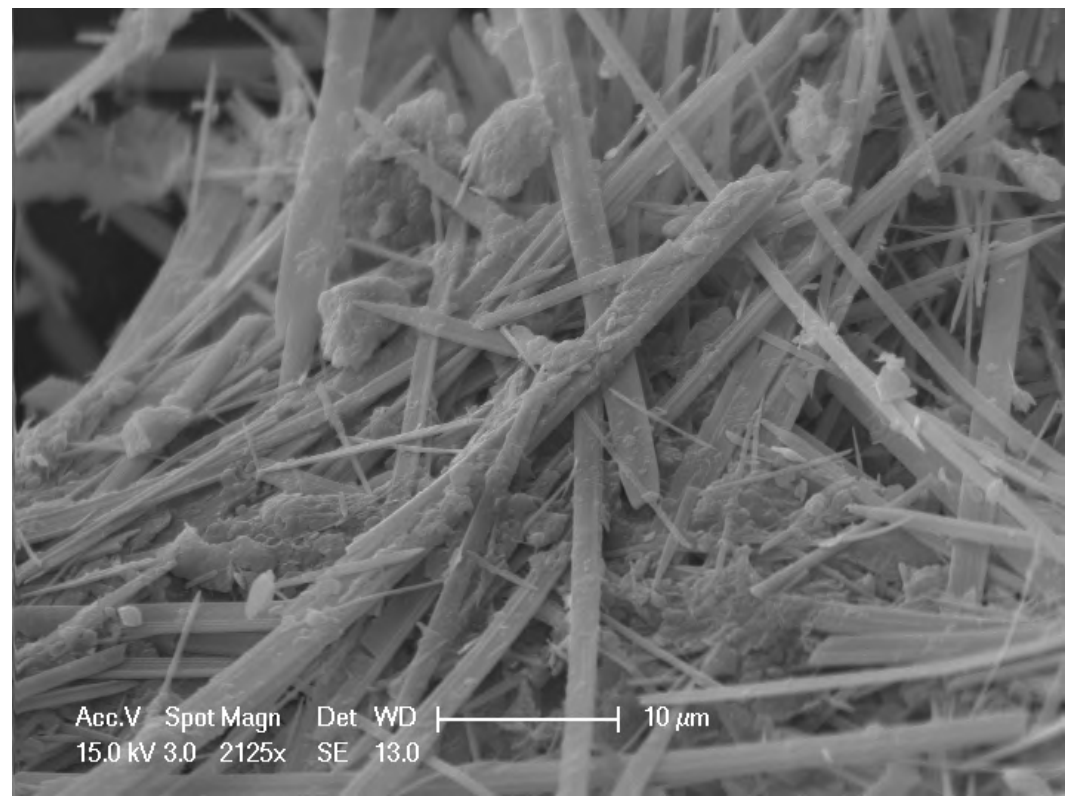

Fig. 8 : Dundasite de Rabotrath (Échantillon RAB-02, photographie au microscope électronique à balayage, mode électrons secondaires).

\subsection{Fluorapatite, $\mathrm{Ca}_{5}\left(\mathrm{PO}_{4}\right)_{3}(\mathrm{~F}, \mathrm{OH}, \mathrm{Cl})$}

Des petits cristaux, observés dans une cavité de blende rubanée, ont été identifiés comme fluorapatite grâce à leur analyse chimique qualitative qui a mis en évidence l'oxygène, le calcium, le phosphore et le fluor comme éléments majeurs. Le microscope électronique à balayage montre de jolis cristaux en barillets hexagonaux, de quelques $10 \mu \mathrm{m}$ de large sur 6 $\mu \mathrm{m}$ de haut. De nombreux cristaux de ce type ont grandi dans une même direction, formant ainsi des plages semblables à des mosaïques (Fig. 9). Sur ces petits cristaux se sont incrustés des petits agglomérats dont l'analyse chimique qualitative montre la présence des éléments $\mathrm{Zn}$ et $\mathrm{S}$; il s'agit donc de sphalérite. Dans la petite géode, on observe également des rhomboèdres d'une trentaine de $\mu \mathrm{m}$ de diamètre, pour lesquels l'analyse chimique qualitative ne révèle que les éléments $\mathrm{Fe}$ et $\mathrm{O}$; il pourrait par conséquent s'agir d'une pseudomorphose de cristaux de sidérite.

Par ailleurs, dans les blocs de brèche à sulfures déjà fortement oxydés en smithsonite, on observe des cavités remplies d'un minéral blanc-beige apparemment argileux. L'analyse par diffraction des rayons $X$ de ce matériel indique qu'il s'agit également d'un minéral du groupe de l'apatite. 


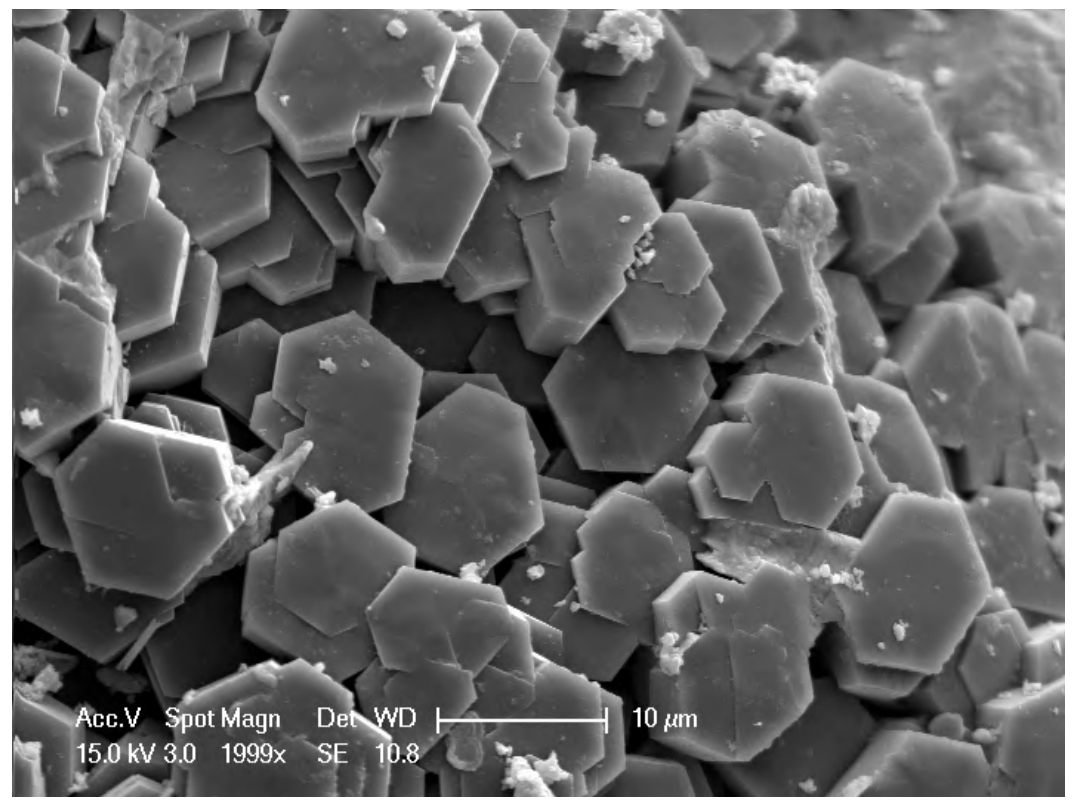

Fig. 9 : Fluorapatite de Rabotrath (Échantillon RAB-14, photographie au microscope électronique à balayage, mode électrons secondaires).

\subsection{Galène, $\mathbf{P b S}$}

La galène est observée en petits cristaux millimétriques fortement corrodés par la cérusite, et déposés sur la schalenblende. La forme cristalline principale est celle de l'octaèdre (Fig. 10). Ce minerai de plomb accompagne habituellement la blende zonée et la marcasite dans les nombreux gisements métalliques exploités jadis par la société «Vieille Montagne » dans l'Est de la Belgique. Comme la marcasite, la galène semble peu abondante à Rabotrath.

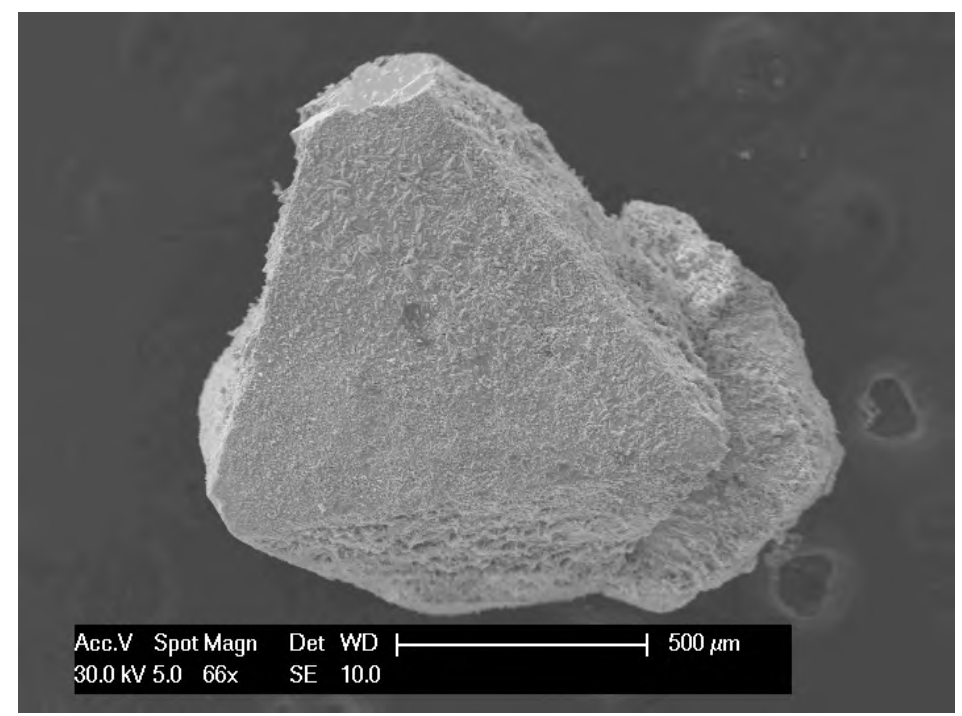

Fig. 10 : Galène de Rabotrath, formant un octaèdre corrodé par la cérusite (Échantillon RAB-

20, photographie au microscope électronique à balayage, mode électrons secondaires). 


\subsection{Gibbsite, $\gamma-\mathrm{Al}(\mathrm{OH}) 3$}

La gibbsite a été identifiée par diffraction des rayons X, en mélange intime avec l'allophane et la zaccagnaite-3R. Sur l'échantillon d'allophane, des parties plus blanches se sont avérées être plus riches en gibbsite. L'analyse chimique qualitative a bien mis en évidence la présence majoritaire des éléments $\mathrm{Al}$ et $\mathrm{O}$, mais également de $\mathrm{Si}$, confirmant ainsi le mélange de gibbsite et d'allophane.

\subsection{Goethite, $\alpha-\mathrm{FeO}(\mathrm{OH})$}

Des masses brunes identifiées visuellement comme de la «limonite », ont été observées parfois en blocs assez volumineux, le long du petit chemin encaissé en contrebas des haldes. L'analyse par diffraction des rayons $X$ a montré qu'il s'agissait de goethite très pure. Sous la loupe binoculaire, dans des petites fentes, on observe parfois de minuscules boules brunes qui, au microscope électronique à balayage, montrent une multitude de petits cristaux de $20 \mu \mathrm{m}$ de long et de $2 \mu \mathrm{m}$ de large (Fig. 11A). La terminaison des cristaux est effilochée (Fig. 11B). L'analyse chimique qualitative montre la seule présence des éléments $\mathrm{Fe}$ et $\mathrm{O}$, confirmant ainsi l'identité du minéral.

A
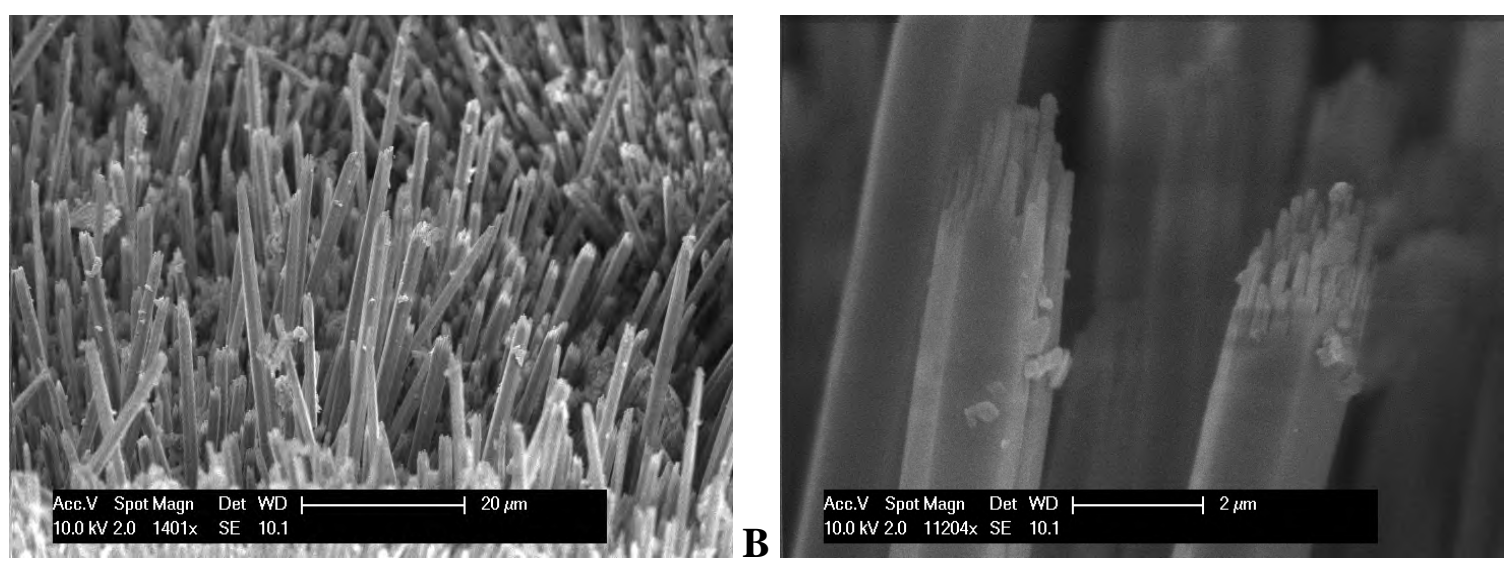

Fig. 11 : Goethite de Rabotrath (Échantillon RAB-18, photographie au microscope électronique à balayage, mode électrons secondaires).

\subsection{Goyazite-plumbogummite, $\left(\mathrm{Sr}, \mathrm{Pb}_{3}\right)_{3}\left(\mathrm{PO}_{4}\right)\left(\mathrm{PO}_{3} \mathrm{OH}\right)(\mathrm{OH})_{6}$}

Un échantillon unique montre, dans des petites cavités, un minéral d'aspect micacé formant des petites boules de couleur crème. Une analyse par diffraction des rayons $\mathrm{X}$ indique que ce minéral appartient au groupe de la crandallite, et un examen au microscope électronique à balayage montre des cristaux en feuillets d'allure grossièrement hexagonale, pouvant atteindre $70 \mu \mathrm{m}$ dans leur plus grande dimension (Fig. 12). Plusieurs spectres EDS ont été réalisés sur ces cristaux ; ils indiquent la présence majoritaire des éléments $\mathrm{O}, \mathrm{Al}, \mathrm{P}, \mathrm{Pb}$ et $\mathrm{Sr}$, avec $\mathrm{Fe}$, $\mathrm{Zn}, \mathrm{Ce}$ et $\mathrm{Ca}$ en plus faibles proportions.

L'affinement des paramètres de maille de ce minéral a été réalisé à l'aide du programme LCLSQ version 8.4 (Burnham, 1991), à partir des valeurs de $d$ mesurées sur le diffractogramme de poudres obtenu au diffractomètre à quatre cercles. Ces paramètres, $a=7,00(1) \AA, c=16,74(7) \AA, \mathrm{V}=710(2) \AA^{3}$, sont intermédiaires entre ceux de la 
plumbogummite (Kolitsch et al., 1999) et de la goyazite (Kato, 1987), indiquant que le minéral de Rabotrath appartient à la solution solide plumbogummite-goyazite. Les rapports $\mathrm{Pb} /(\mathrm{Pb}+\mathrm{Sr})$, obtenus à partir des analyses EDS semi-quantitatives, sont compris entre 0,40 et 0,50 , ce qui montre que le minéral est très proche de la limite entre les deux espèces, mais semblerait plutôt correspondre à une goyazite riche en plomb. Plumbogummite et goyazite sont des minéraux nouveaux pour la Belgique.

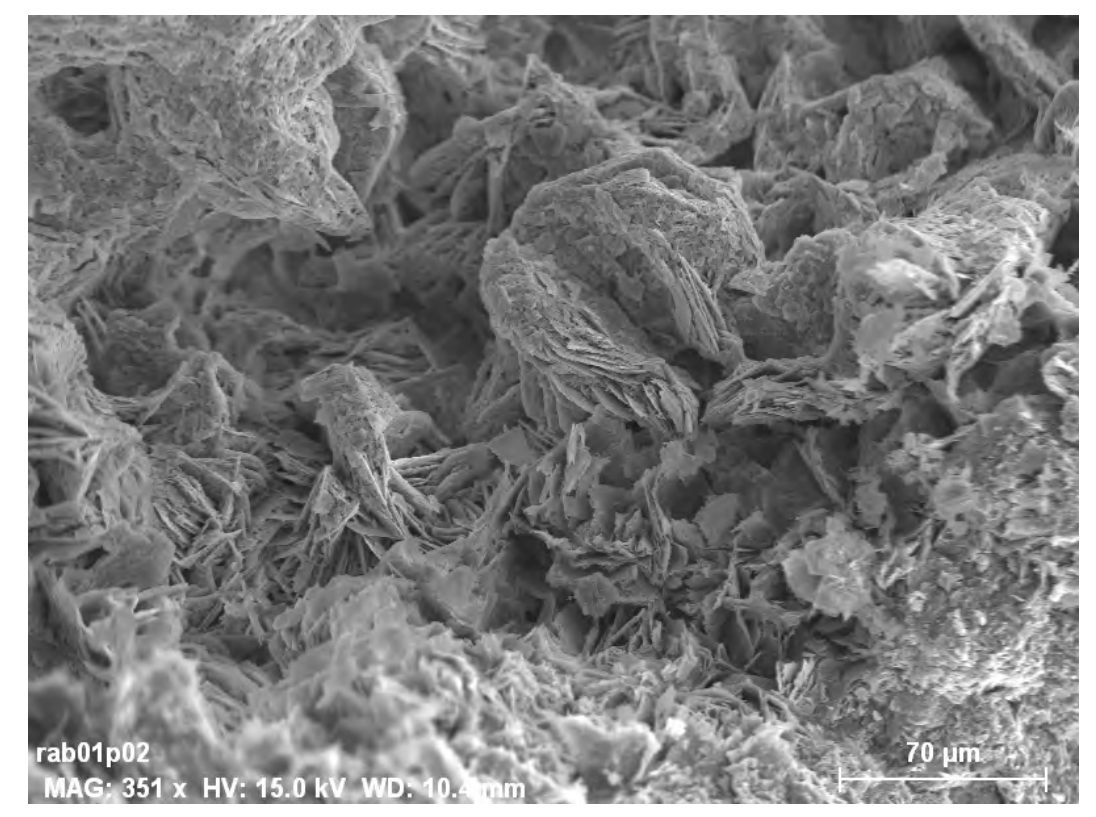

Fig. 12 : Agrégats de tablettes de goyazite-plumbogummite de Rabotrath (Échantillon RAB-

01, photographie au microscope électronique à balayage, mode électrons secondaires).

\subsection{Gypse, $\mathrm{CaSO}_{4} \cdot 2 \mathrm{H}_{2} \mathrm{O}$}

Des petits cristaux observés à la surface de blocs riches en sphalérite et marcasite sont visuellement identifiés comme étant du gypse. Le spectre EDS indique la présence des éléments $\mathrm{Ca}, \mathrm{S}$ et $\mathrm{O}$, confirmant ainsi l'identification du minéral. Chaque cristal de gypse ne dépasse pas $50 \mu \mathrm{m}$ de longueur mais, associés en grand nombre, ils forment des plages de plusieurs $\mathrm{mm}^{2}$ (Fig. 13). Ce minéral secondaire est connu pour se former rapidement dans les terrils contenant des blocs minéralisés en sulfures et percolés par les eaux de pluie.

De minuscules fragments assez informes sont observés nombreux à la surface des cristaux de gypse. L'analyse chimique qualitative montre la présence majoritaire du zinc et du soufre, ainsi que du fer fort minoritaire ; il s'agit donc de sphalérite. 


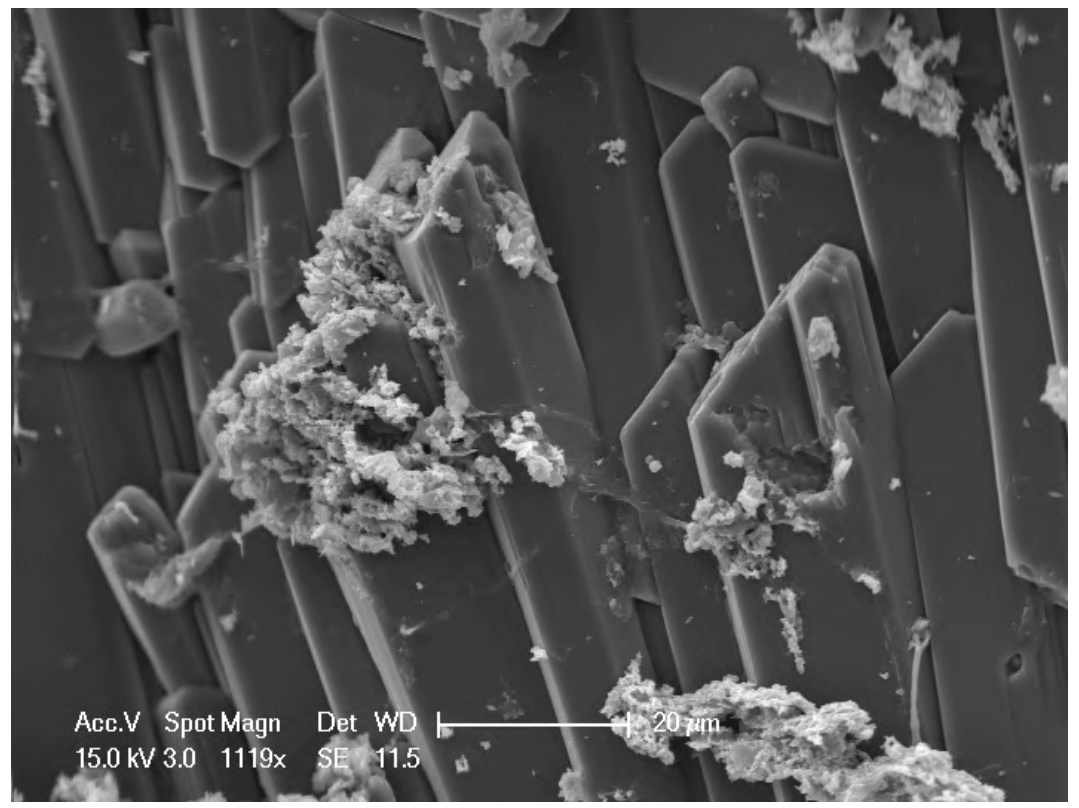

Fig. 13 : Agrégat de cristaux de gypse de Rabotrath, montrant des terminaisons caractéristiques. Le minéral granulaire déposé sur le gypse est constitué de sphalérite (Échantillon RAB-25, photographie au microscope électronique à balayage, mode électrons secondaires).

\subsection{Hawleyite, CdS}

Des enduits jaunes de taille millimétrique sont parfois observés sur la sphalérite rubanée. L'analyse par diffraction des rayons $\mathrm{X}$, réalisée au diffractomètre à quatre cercles en mode «Debye-Scherrer », confirme qu'il s'agit bien de hawleyite, et non de son polymorphe la greenockite. Ce minéral est un produit d'altération classique des sphalérites cadmifères (Hatert et al., 2002).

\subsection{Hematite, $\mathrm{Fe}_{2} \mathrm{O}_{3}$}

Des nodules de sidérite massive sont parfois entourés d'une auréole rouge d'éclat terreux. L'analyse par diffraction des rayons X confirme l'identification d'hématite.

\subsection{Marcasite, $\mathrm{FeS}_{2}$}

La marcasite forme de petits nodules dans lesquels se trouvent des petites cavités remplies de cristaux rhomboédriques de sidérite, dont la couleur varie du vert pâle au brun. L'identification est confirmée par diffraction des RX. Des petits cristaux parfois maclés d'une centaine de $\mu \mathrm{m}$ de longueur ont été observés dans une petite géode (Fig. 14) ; leur analyse chimique qualitative met en évidence la présence majoritaire du fer et du soufre. La marcasite est un minéral accompagnant fréquemment la blende zonée et la galène dans les gisements $\mathrm{Pb}-\mathrm{Zn}$ exploités anciennement en Belgique. À Rabotrath, elle semble cependant assez peu fréquente. 


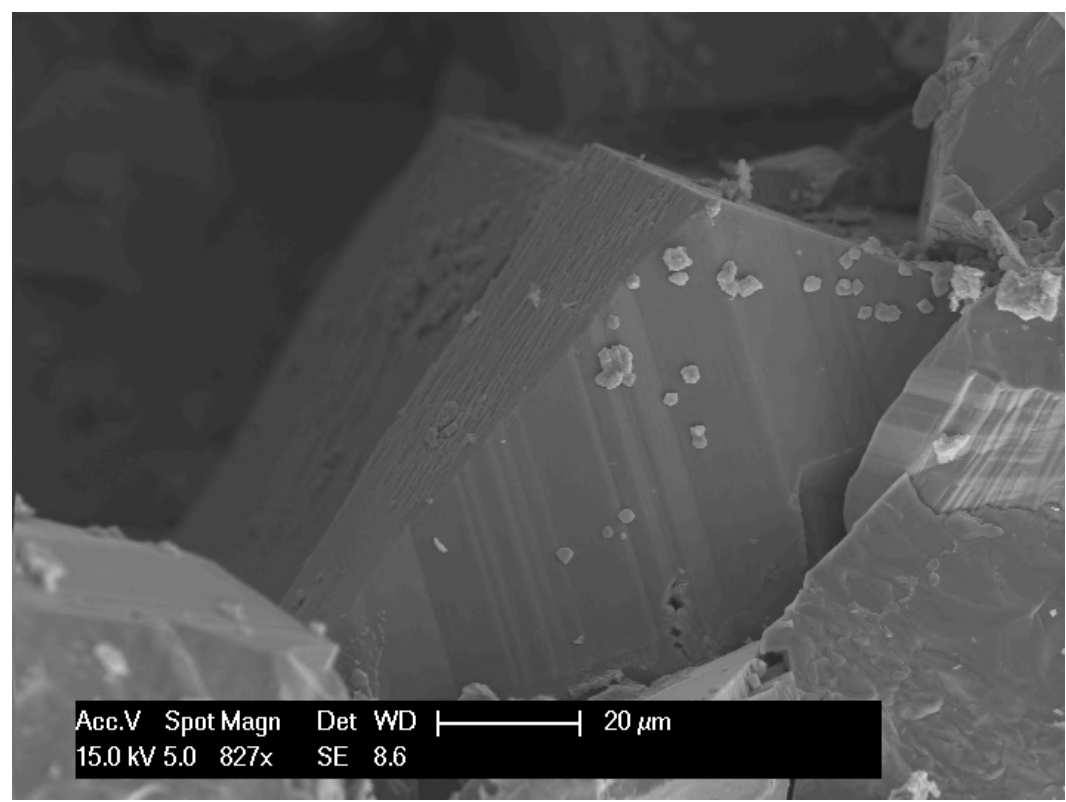

Fig. 14 : Cristal de marcasite de Rabotrath (Échantillon RAB-21, photographie au microscope électronique à balayage, mode électrons secondaires).

\subsection{Parascholzite, $\mathrm{CaZn}_{2}\left(\mathrm{PO}_{4}\right)_{2} \cdot 2 \mathrm{H}_{2} \mathrm{O}$}

Un nodule atteignant $5 \mathrm{~cm}$ de diamètre est entièrement constitué d'un minéral incolore à blanc, formant de petits cristaux en aiguille et des agrégats fibro-radiés au sein de cavités (Fig. 15). L'analyse par diffraction des rayons $\mathrm{X}$ montre un diffractogramme de poudres semblable à celui de la parascholzite de Hagendorf, Allemagne (Tableau 1). Une comparaison avec le diffractogramme de poudres de la scholzite de Reaphook Hill, Australie, montre une bien moins bonne correspondance. L'analyse chimique qualitative confirme les éléments $\mathrm{Ca}$, $\mathrm{Zn}$, $\mathrm{P}$ et $\mathrm{O}$. La parascholzite est une espèce minérale rare, qui n'était pas encore recensée en Belgique.

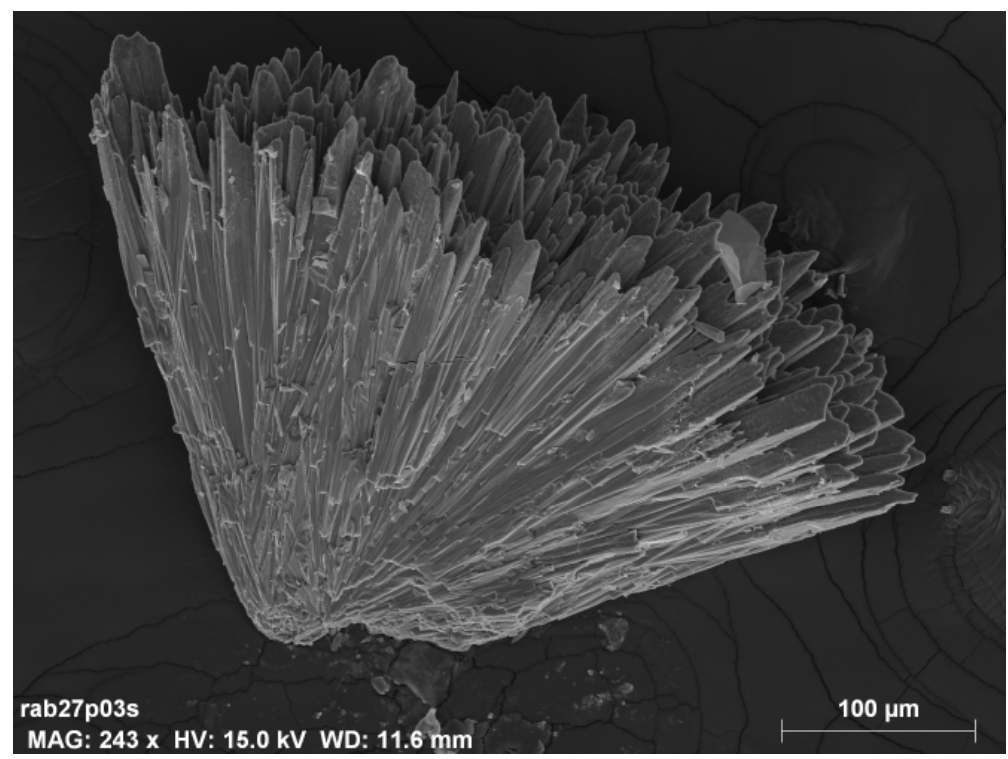

Fig. 15 : Agrégat de cristaux en aiguille de parascholzite, Rabotrath (Échantillon RAB-27, photographie au microscope électronique à balayage, mode électrons secondaires). 


\subsection{Plumbojarosite, $\mathrm{Pb}_{0,5} \mathrm{Fe}_{3}\left(\mathrm{SO}_{4}\right)_{2}(\mathrm{OH})_{6}$}

Des croûtes brunes, visuellement identifiées comme constituées d'un matériel jarositique, ont été observées sur un échantillon de marcasite (Fig. 16). Un échantillon unique montre également une cavité contenant des cristaux brun-orange identifiés comme un mineral jarositique (Fig. 17). Une analyse par diffraction des rayons $X$ montre qu'il s'agit vraisemblablement de plumbojarosite, ce que confirme le spectre EDS. Le fer, le soufre, l'oxygène et le plomb sont les éléments majoritaires. Le potassium, bien que très minoritaire par rapport au plomb, est également détecté, indiquant que cette phase jarositique est une solution solide entre la jarosite et la plumbojarosite. Rabotrath est la seconde occurrence belge pour la plumbojarosite, qui n'était connue qu'à La Roche-en-Ardenne (Blondieau \& Hatert, 2009).

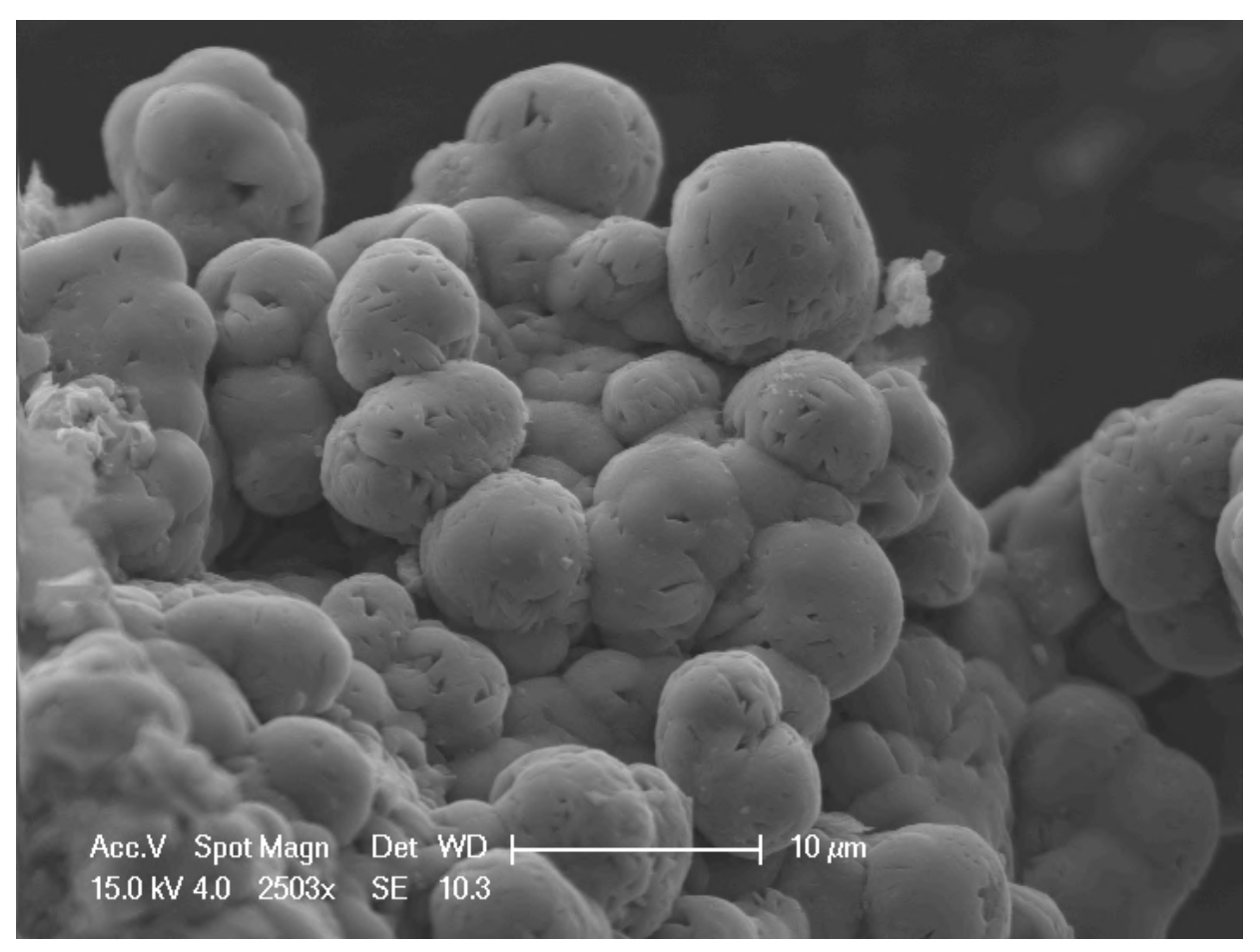

Fig. 16 : Petites sphérules de plumbojarosite de Rabotrath (Échantillon RAB-10, photographie au microscope électronique à balayage, mode électrons secondaires). 
Bulletin de la Société Royale des Sciences de Liège, Vol. 85, 2016, p. 30 - 55

Tableau 1 : Diffractogramme de poudres de rayons X de la parascholzite de Rabotrath.

\begin{tabular}{|c|c|c|c|c|c|}
\hline \multicolumn{2}{|c|}{$\begin{array}{l}\text { Parascholzite } \\
\text { Rabotrath }\end{array}$} & \multicolumn{2}{|c|}{$\begin{array}{c}\text { Parascholzite }^{*} \\
\text { Hagendorf, } \\
\text { Allemagne } \\
\end{array}$} & \multicolumn{2}{|c|}{$\begin{array}{c}\text { Scholzite }^{* *} \\
\text { Reaphook Hill, } \\
\text { Australie } \\
\end{array}$} \\
\hline$d(\AA)$ & $\mathrm{I} / \mathrm{I}_{0}(\%)$ & $d(\AA)$ & $\mathrm{I} / \mathrm{I}_{0}(\%)$ & $d(\AA)$ & $\mathrm{I} / \mathrm{I}_{0}(\%)$ \\
\hline- & - & - & - & 9,3 & 15 \\
\hline 8,56 & 100 & 8,55 & 100 & 8,65 & 100 \\
\hline- & - & - & - & 7,66 & 5 \\
\hline 6,81 & 5 & 6,80 & 10 & 6,92 & 5 \\
\hline 4,95 & 1 & 4,95 & 5 & - & - \\
\hline 4,52 & 23 & 4,53 & 30 & 4,56 & 25 \\
\hline 4,43 & 2 & 4,424 & 5 & - & - \\
\hline 4,28 & 11 & 4,287 & 10 & 4,29 & 40 \\
\hline 4,16 & 11 & 4,158 & 50 & 4,16 & 5 \\
\hline- & - & - & - & 3,75 & 5 \\
\hline 3,71 & 8 & 3,709 & 10 & 3,71 & 20 \\
\hline 3,41 & 21 & 3,406 & 40 & 3,40 & 30 \\
\hline 3,36 & 8 & 3,360 & 10 & 3,34 & 5 \\
\hline- & - & 3,313 & 1 & - & - \\
\hline 3,21 & 2 & 3,207 & 5 & 3,25 & 10 \\
\hline 3,16 & 6 & 3,153 & 20 & 3,18 & 10 \\
\hline 3,11 & 10 & 3,109 & 10 & 3,12 & 20 \\
\hline 3,01 & 3 & 3,005 & 10 & 3,03 & 5 \\
\hline 2,947 & 1 & 2,945 & 5 & - & - \\
\hline 2,860 & 1 & - & - & 2,865 & 15 \\
\hline 2,805 & 52 & 2,804 & 80 & 2,806 & 60 \\
\hline 2,781 & 9 & 2,779 & 40 & - & - \\
\hline- & - & 2,766 & 5 & - & - \\
\hline 2,752 & 4 & 2,752 & 10 & - & - \\
\hline 2,687 & 1 & - & - & 2,687 & 15 \\
\hline- & - & - & - & 2,640 & 5 \\
\hline- & - & - & - & 2,633 & 10 \\
\hline 2,587 & 5 & 2,586 & 30 & 2,584 & 10 \\
\hline 2,553 & 2 & 2,553 & 1 & - & - \\
\hline 2,514 & 1 & 2,511 & 1 & - & - \\
\hline 2,479 & 3 & 2,475 & 10 & 2,482 & 20 \\
\hline 2,425 & 2 & 2,424 & 5 & - & - \\
\hline- & - & - & - & 2,398 & 5 \\
\hline - & - & - & - & 2,390 & 5 \\
\hline 2,374 & 2 & 2,374 & 1 & 2,382 & 10 \\
\hline- & - & 2,332 & 5 & - & - \\
\hline 2,326 & 9 & 2,325 & 5 & 2,327 & 25 \\
\hline 2,303 & 1 & 2,299 & 1 & - & - \\
\hline 2,263 & 17 & 2,263 & 20 & 2,266 & 30 \\
\hline 2,212 & 1 & 2,212 & 5 & - & - \\
\hline 2,180 & 1 & 2,180 & 1 & - & - \\
\hline- & - & - & - & 2,150 & 5 \\
\hline 2,082 & 1 & 2,082 & 1 & 2,080 & 10 \\
\hline 2,006 & 4 & 2,005 & 5 & 2,000 & 20 \\
\hline 1,991 & 3 & 1,989 & 5 & - & - \\
\hline 1,908 & 3 & 1,908 & 20 & - & - \\
\hline 1,832 & 2 & 1,829 & 1 & 1,840 & 5 \\
\hline 1,814 & 9 & 1,814 & 20 & 1,810 & 15 \\
\hline
\end{tabular}




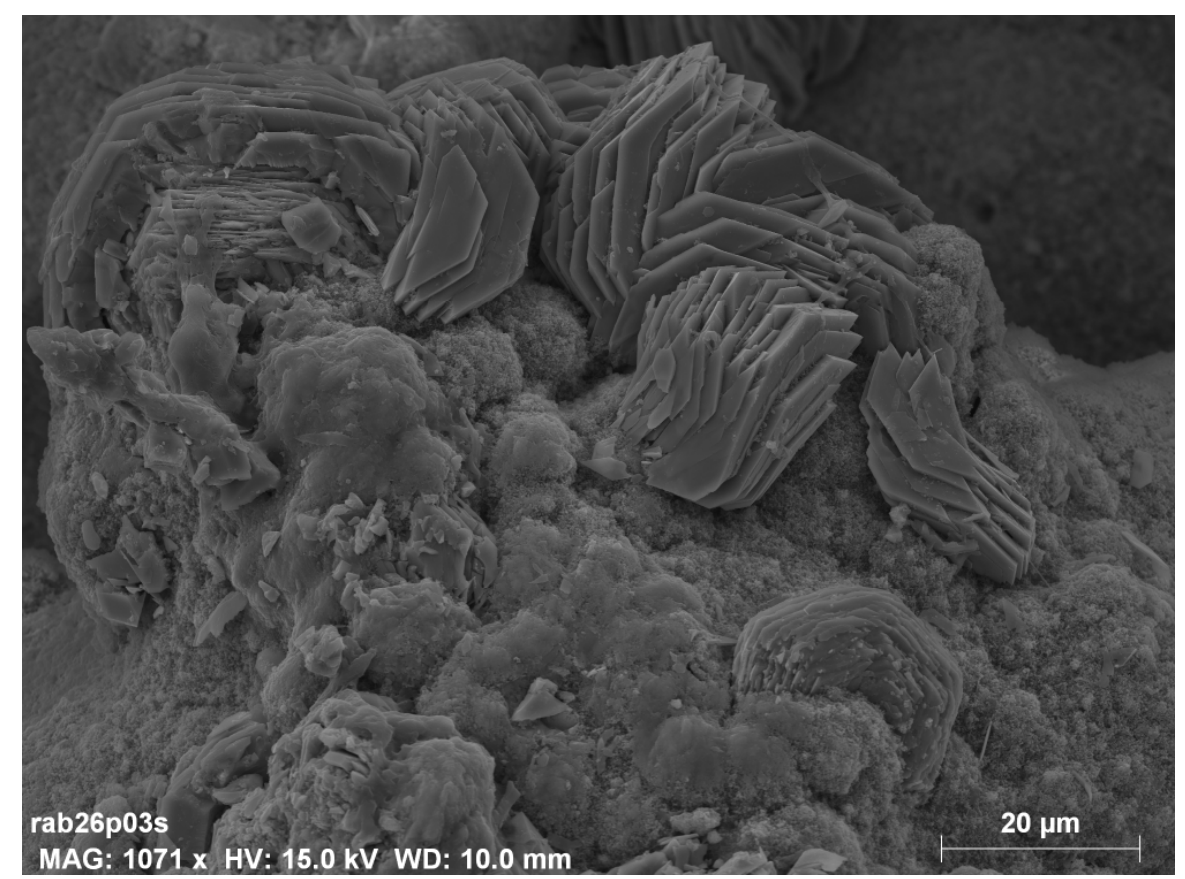

Fig. 17 : Cristaux de plumbojarosite de Rabotrath (Échantillon RAB-26, photographie au microscope électronique à balayage, mode électrons secondaires).

\subsection{Pyromorphite, $\mathrm{Pb}_{5}\left(\mathrm{PO}_{4}\right)_{3} \mathrm{Cl}$}

Des petites plages blanc rosé d'aspect gras, tapissant de petites géodes, montrent de minuscules cristaux visuellement identifiées comme pyromorphite par comparaison avec des échantillons de Schimper (Blondieau \& Polrot, 2011). De la pyromorphite en globules et petits cristaux de couleur vert pomme a aussi été observée sur des plages de smithsonite noyées dans de la sidérite brune. Le microscope électronique à balayage montre des cristaux prismatiques de section hexagonale terminés par une pyramide, ou des agrégats de cristaux en aiguilles (Fig. 18). L'analyse chimique qualitative, réalisée par EDS sur ces cristaux dont la taille varie entre 10 et $40 \mu \mathrm{m}$, indique bien la présence majoritaire des éléments $\mathrm{Pb}, \mathrm{P}, \mathrm{O}$ et $\mathrm{Cl}$, avec $\mathrm{Ca}$ en plus faibles proportions. Dans les petites géodes, la pyromorphite est associée à des cristaux de smithsonite. Dans des petites fissures parcourant la blende zonée, le minéral se présente en cristaux blanc ne dépassant pas $20 \mu \mathrm{m}$ de longueur, associés à des rhomboèdres de sidérite. 


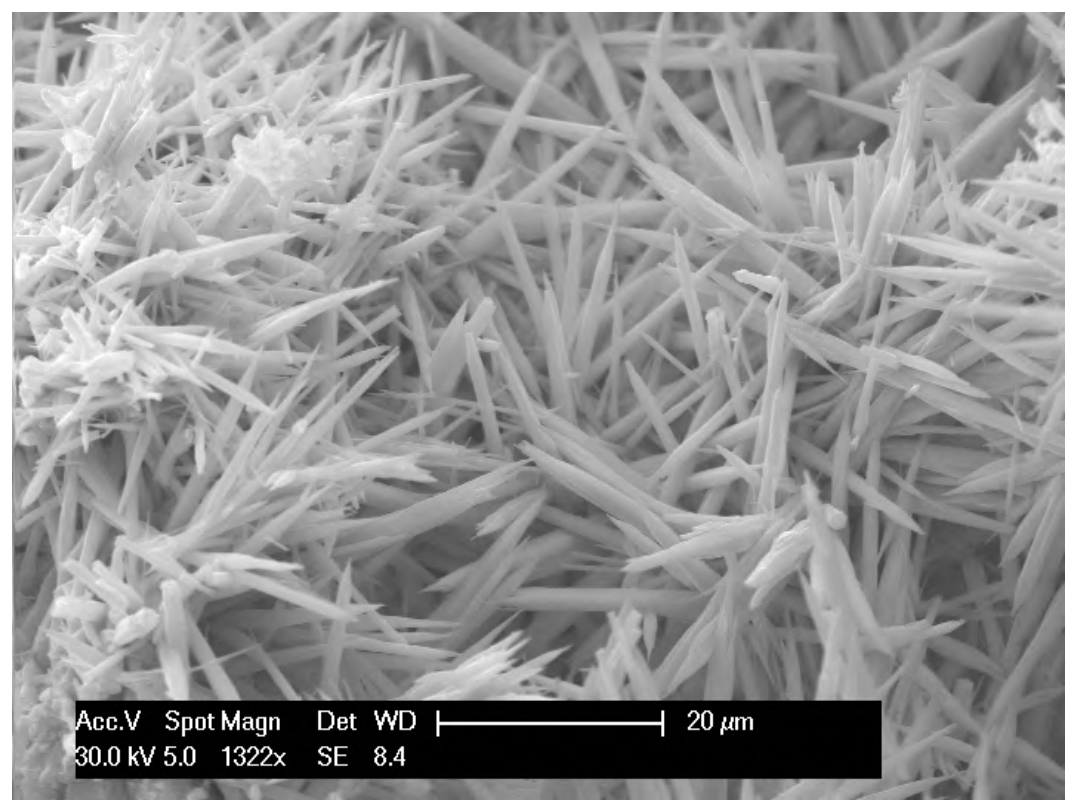

Fig. 18 : Petites aiguilles de pyromorphite, Rabotrath (Échantillon RAB-19, photographie au microscope électronique à balayage, mode électrons secondaires).

\subsection{Quartz, $\mathrm{SiO}_{2}$}

Le quartz n'a pas été observé en cristaux automorphes; visuellement, il se présente en minuscules fragments associés à la «calamine ». Un échantillon de cette «calamine », duquel avaient été soigneusement retirés tous les fragments de quartz, a été analysé par diffraction des rayons $\mathrm{X}$, mais le quartz s'est néanmoins encore révélé être associé à la smithsonite. Ceci indique que le minéral est présent en particules de très petite taille dans les masses « calaminaires ».

\subsection{Sidérite, $\mathrm{FeCO}_{3}$}

À Rabotrath, les différents minerais sulfurés, «schalenblende» et marcasite, s'observent fréquemment en fragments de dimensions variables, cimentés dans une brèche. Une analyse par diffraction des rayons $\mathrm{X}$, réalisée sur le ciment gris-vert à brun foncé de cette brèche, montre qu'il est majoritairement constitué de sidérite, avec un peu de goethite minoritaire. Le spectre EDS confirme les éléments $\mathrm{Fe}, \mathrm{C}$ et $\mathrm{O}$ dominants.

Par ailleurs, des petits rhomboèdres vert pâle à bruns, d'une centaine de $\mu \mathrm{m}$ de diamètre, sont observés dans des cavités de marcasite (Fig. 19). Leur spectre EDS confirme la sidérite, avec les éléments principaux $\mathrm{Fe}, \mathrm{C}$, O, ainsi que $\mathrm{Ca}$ et $\mathrm{Zn}$ minoritaires. L'examen au microscope électronique à balayage a montré que la sidérite était parfois associée à de petits cristaux d'anglésite ou de pyromorphite. 


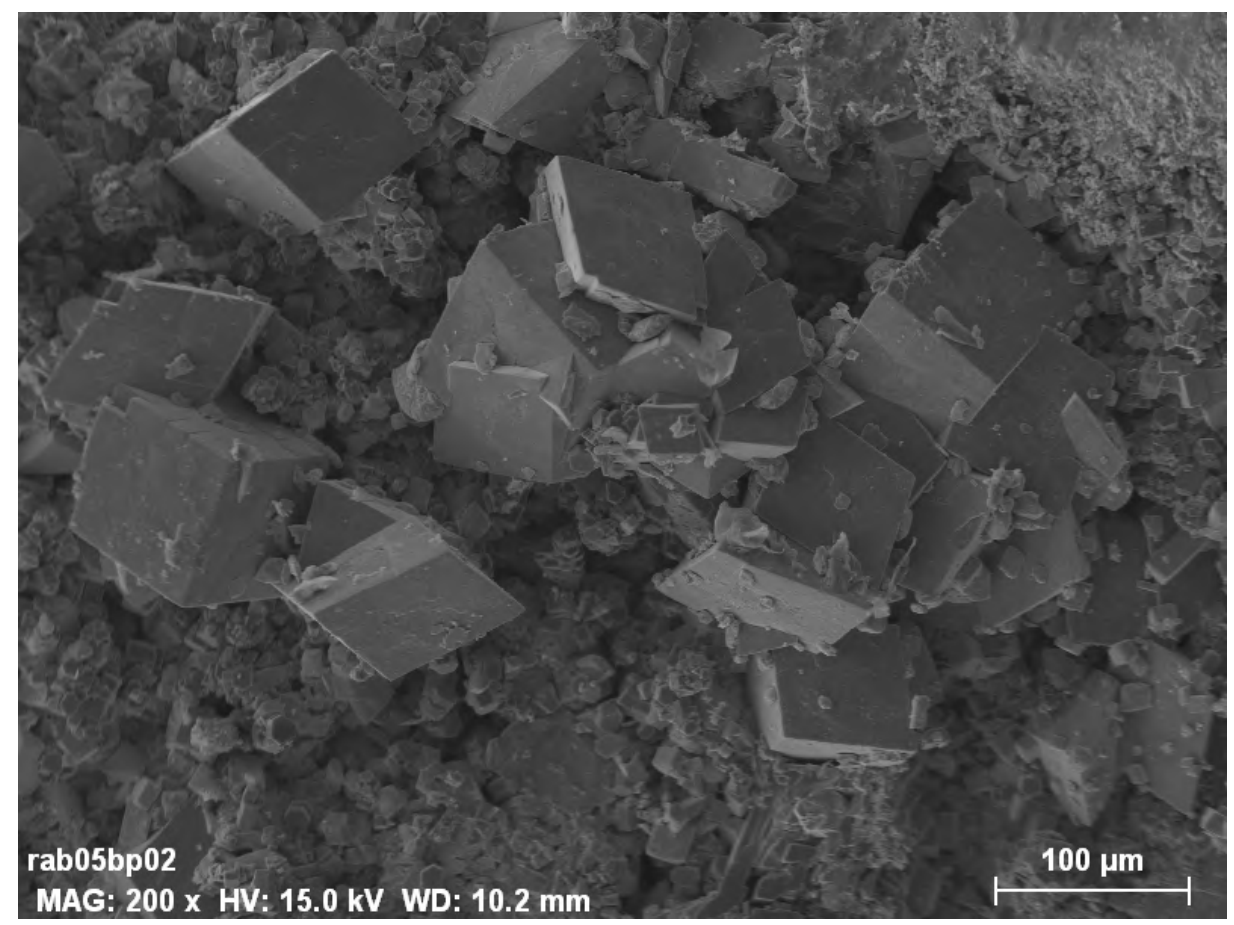

Fig. 19 : Rhomboèdres de sidérite de Rabotrath (Échantillon RAB-05, photographie au microscope électronique à balayage, mode électrons secondaires).

\subsection{Smithsonite, $\mathrm{ZnCO}_{3}$}

La «calamine » exploitée à Rabotrath était essentiellement composée de smithsonite. Ce minéral est encore très fréquent sur le site, en petits fragments ou le plus souvent sous forme de «calamine roche », c'est-à-dire des masses beiges micro-bréchiques dont le ciment est formé de smithsonite. Les éléments de la brèche sont des grains de sable, des fragments d'argile, des petits fragments arrondis de «limonite »; le tout est parfois teinté de noir par la chalcophanite.

Sur certains échantillons, il est possible d'observer de la smithsonite très pure. Elle est alors très claire, presque blanche en surface mais gris bleutée sur cassure. Elle est constituée d'une multitude de petites couches de quelques millimètres d'épaisseur, qui s'empilent les unes sur les autres. Parfois aussi des cristaux d'aspect arrondis sont visuellement observés dans des cavités se trouvant dans les parties les plus bleutées. Plus rarement, la smithsonite s'observe sous une forme concrétionnée gris-bleutée et peut alors fournir des échantillons très esthétiques (Fig. 20A).

Des blocs très oxydés de brèche à sulfures, cimentés par la sidérite, montrent parfois des cavités tapissées de smithsonite dans laquelle subsistent parfois des reliques de sphalérite. Les plages de smithsonite blanche, cimentées dans la sidérite brune, ressemblent un peu aux coquilles d'une lumachelle.

Des analyses par diffraction des rayons $\mathrm{X}$ ont été réalisées sur un échantillon bien concrétionné gris-bleu, ainsi que sur un échantillon de «calamine-roche ». Dans les deux cas, 
il s'agit de smithsonite, avec également un peu de quartz dans la «calamine-roche ». Des couches blanches d'aspect plus «spongieux» alternent parfois avec les couches grisbleutées ; leur analyse par diffraction des rayons X a confirmé l'identification de smithsonite.

Les cristaux visuellement observés comme arrondis montrent, en microscopie électronique à balayage, une morphologie rhomboédrique (Figs. 20B à D). Leur taille est de l'ordre d'une vingtaine de $\mu \mathrm{m}$, et les spectres EDS indiquent les éléments $\mathrm{Zn}, \mathrm{C}$ et $\mathrm{O}$ majoritaires, avec parfois Fe toujours très minoritaire. La smithsonite en cristaux de morphologie scalénoédrique est également observée en association avec la pyromorphite.
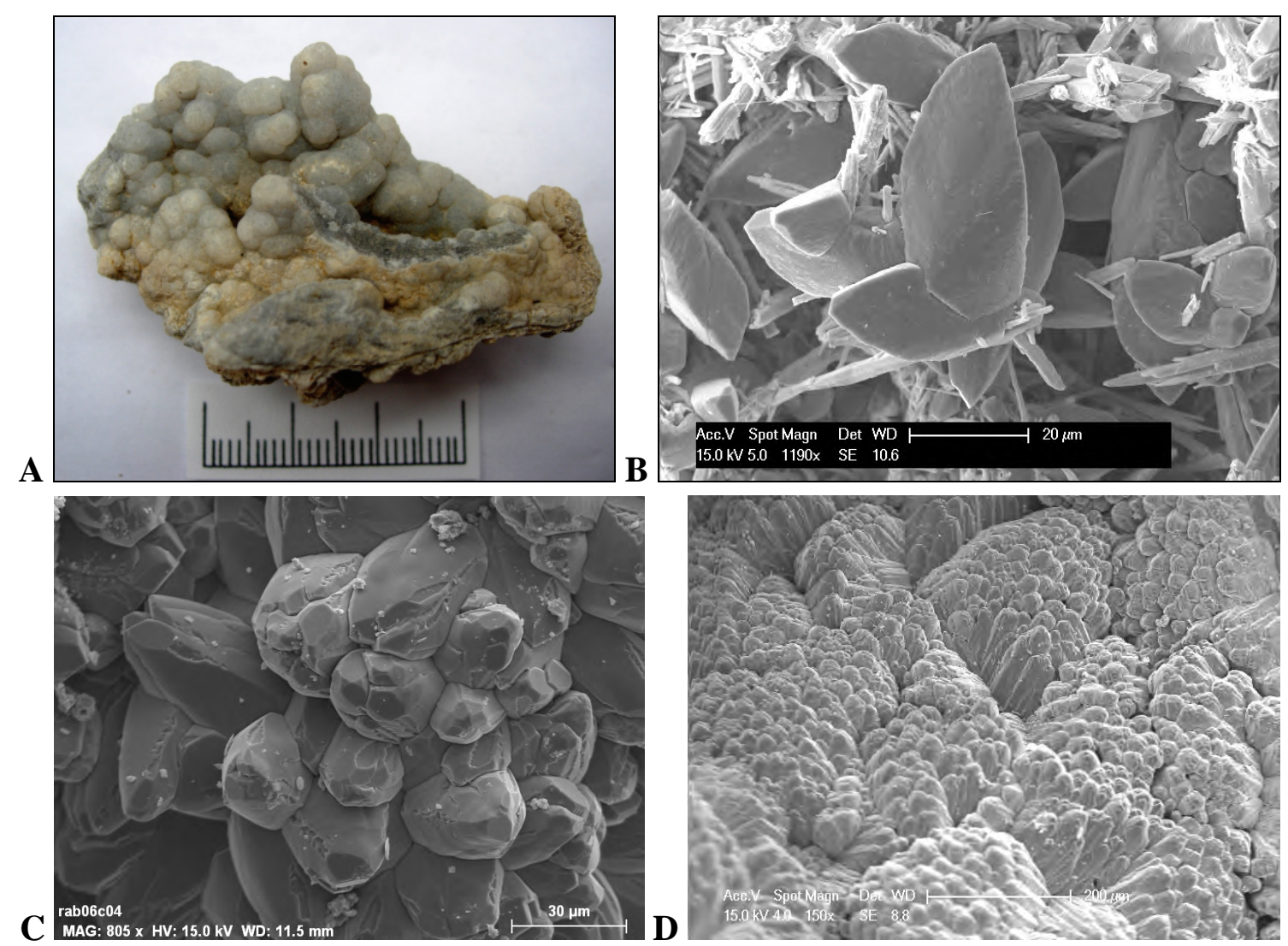

Fig. 20 : Différents faciès de la smithsonite de Rabotrath (B, C, D : photographies au microscope électronique à balayage, mode électrons secondaires. $\mathrm{B}: \mathrm{RAB}-23$; C et D : RAB-06).

\subsection{Soufre, $S$}

De minuscules cristaux jaunes, d'une taille inférieure à $50 \mu \mathrm{m}$, sont observés dans de petites cavités de la blende zonaire. Visuellement, ils sont identifiés comme du soufre ; l'analyse chimique qualitative à l'EDS confirme $\mathrm{S}$ comme seul élément. Le soufre est un minéral fréquent dans les haldes à sulfures, mais il passe souvent inaperçu du fait de sa petite taille et de sa couleur jaune souvent très pâle. C'est un minéral qui se forme rapidement dans les déblais riches en sulfures, percolés par les eaux météoriques. 


\subsection{Sphalérite, $\mathrm{ZnS}$}

Comme dans la plupart des autres sites du district métallifère $\mathrm{Pb}-\mathrm{Zn}$ de l'est de la Belgique, le minerai de zinc sulfuré de Rabotrath se présente sous forme de «blende rubanée » appelée aussi «blende zonée » ou «schalenblende ». Généralement, ce minerai rubané est constitué d'une alternance de couches de sphalérite collomorphe de couleur variable, allant du beige clair au noir. À Rabotrath, toutefois, le minerai est souvent beige, crème ou brun très clair, voire blanc et fort peu zoné. Cette teinte très claire est surprenante, en comparaison aux échantillons provenant des autres gisements du district. Le spectre EDS, obtenu sur un fragment bréchique crème clair de cette sphalérite, montre la présence majoritaire de $\mathrm{Zn}$ et $\mathrm{S}$, avec une teneur en Fe très faible. Une analyse semi-quantitative indique moins de $1 \%$ en poids Fe.

Une analyse par diffraction des rayons $\mathrm{X}$ de ce minerai très clair montre qu'il est uniquement constitué de sphalérite, et qu'il n'y a pas de würtzite associée. La littérature signale en effet que certaines blendes zonées sont un mélange de sphalérite et de würtzite ; cela ne semble pas être le cas à Rabotrath.

La blende plus brune est également présente à Rabotrath, mais moins fréquente. Elle semble cependant plus cristalline et de minuscules cristaux jaunâtres de sphalérite s'observent parfois dans de petites cavités. Les rognons de sphalérite, souvent blancs en surface, sont inclus dans une brèche brun-rouge dont le ciment est principalement constitué de sidérite (Fig. 21). Il s'agit de la « sidérose » signalée dans les textes anciens.

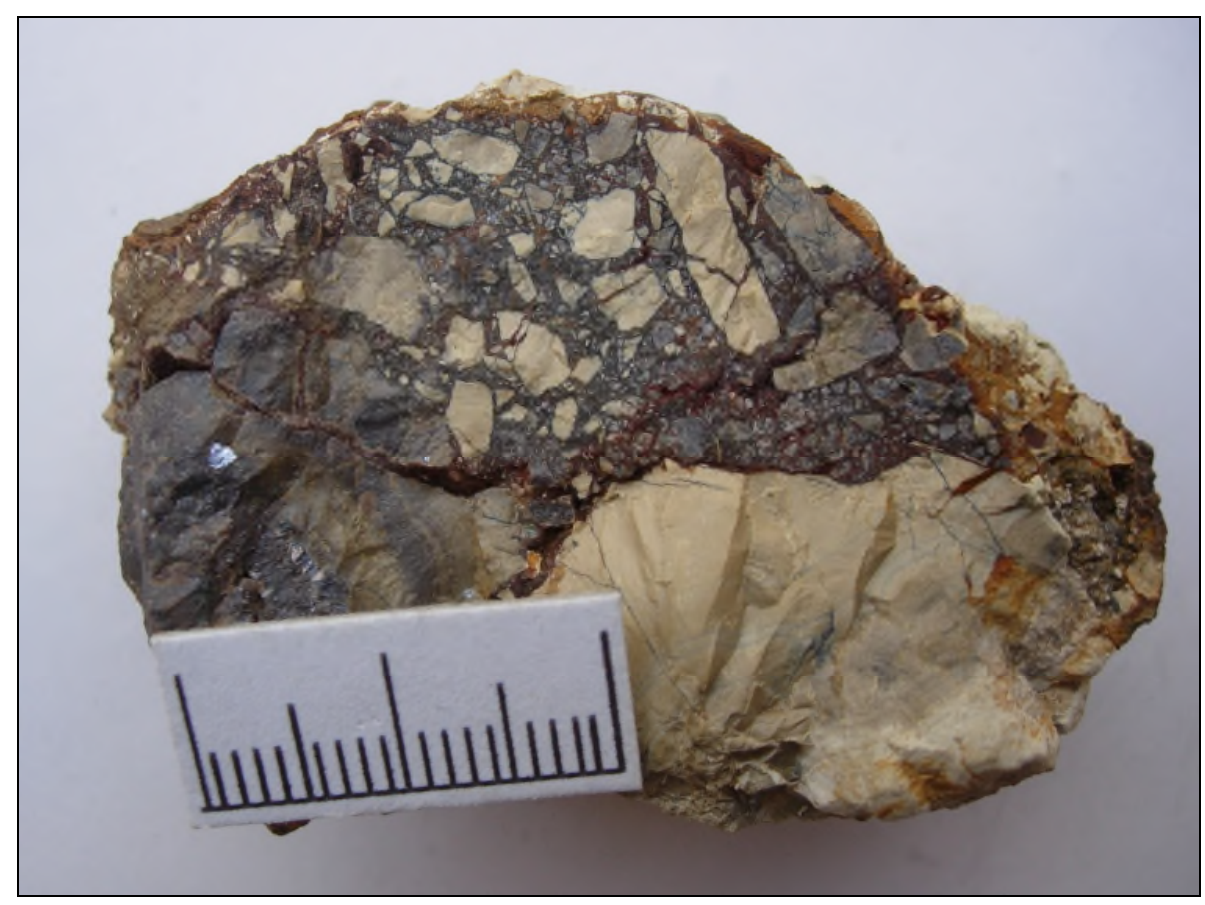

Fig. 21 : Brèche à sulfures (sphalérite très claire majoritaire) cimentée par de la sidérite gris verdâtre, Rabotrath. 


\subsection{Zaccagnaite-3R $\mathrm{Zn}_{4} \mathrm{Al}_{2}(\mathrm{OH})_{12} \mathrm{CO}_{3} .3 \mathrm{H}_{2} \mathrm{O}$}

Le minéral est observé en agrégats centimétriques constitués de petites paillettes blanches, parfois blanc-neige, d'aspect micacé. Dans de petites géodes, il se présente en sphérules d'un diamètre inférieur à $100 \mu \mathrm{m}$ (Fig. 22), le plus souvent associées à de l'allophane vitreuse. Deux échantillons ont été analysés par diffraction des rayons $\mathrm{X}$ sur poudres; ils montrent la présence de zaccagnaite-3R associée à la gibbsite. Quelques pics pourraient également correspondre à ceux de la fraipontite (Tableau 2).

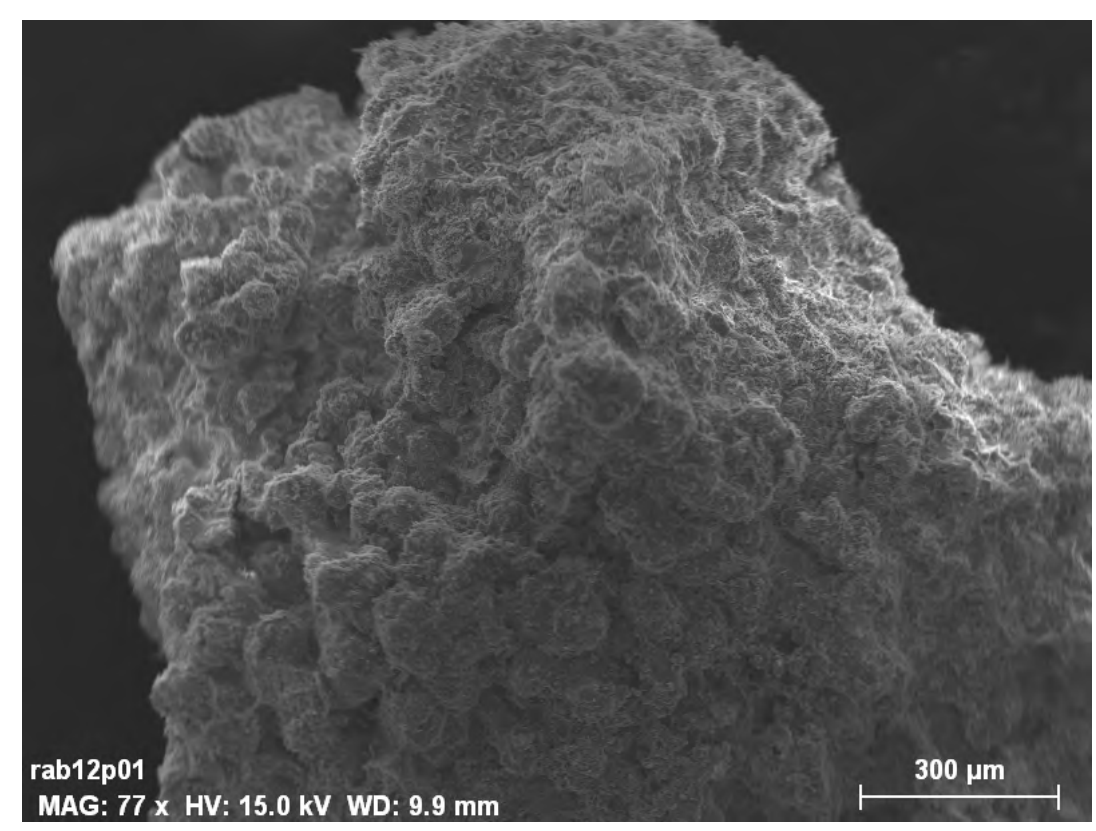

Fig. 22 : Zaccagnaite-3R, Rabotrath (Échantillon RAB-12 ; photographie au microscope électronique à balayage, mode électrons secondaires).

Les spectres EDS mettent en évidence la présence des éléments $\mathrm{Al}, \mathrm{Zn}, \mathrm{C}$ et $\mathrm{O}$ mais également un peu de $\mathrm{Si}$ et de Fe (Fig. 23). L'allophane, un minéral amorphe observé visuellement en association avec la zaccagnaite, pourrait expliquer la présence de silicium détectée lors de l'analyse chimique qualitative.

Un autre échantillon montre de belles mais minuscules croûtes blanc neige, ainsi que des boules blanches à écailles nacrées dans de la smithsonite, qui ont été identifiées comme zaccagnaite-3R par diffraction des rayons $\mathrm{X}$ et spectrométrie EDS. 
Bulletin de la Société Royale des Sciences de Liège, Vol. 85, 2016, p. 30 - 55

Tableau 2 : Diffractogrammes de poudres de rayons X de la zaccagnaite-3R de Rabotrath.

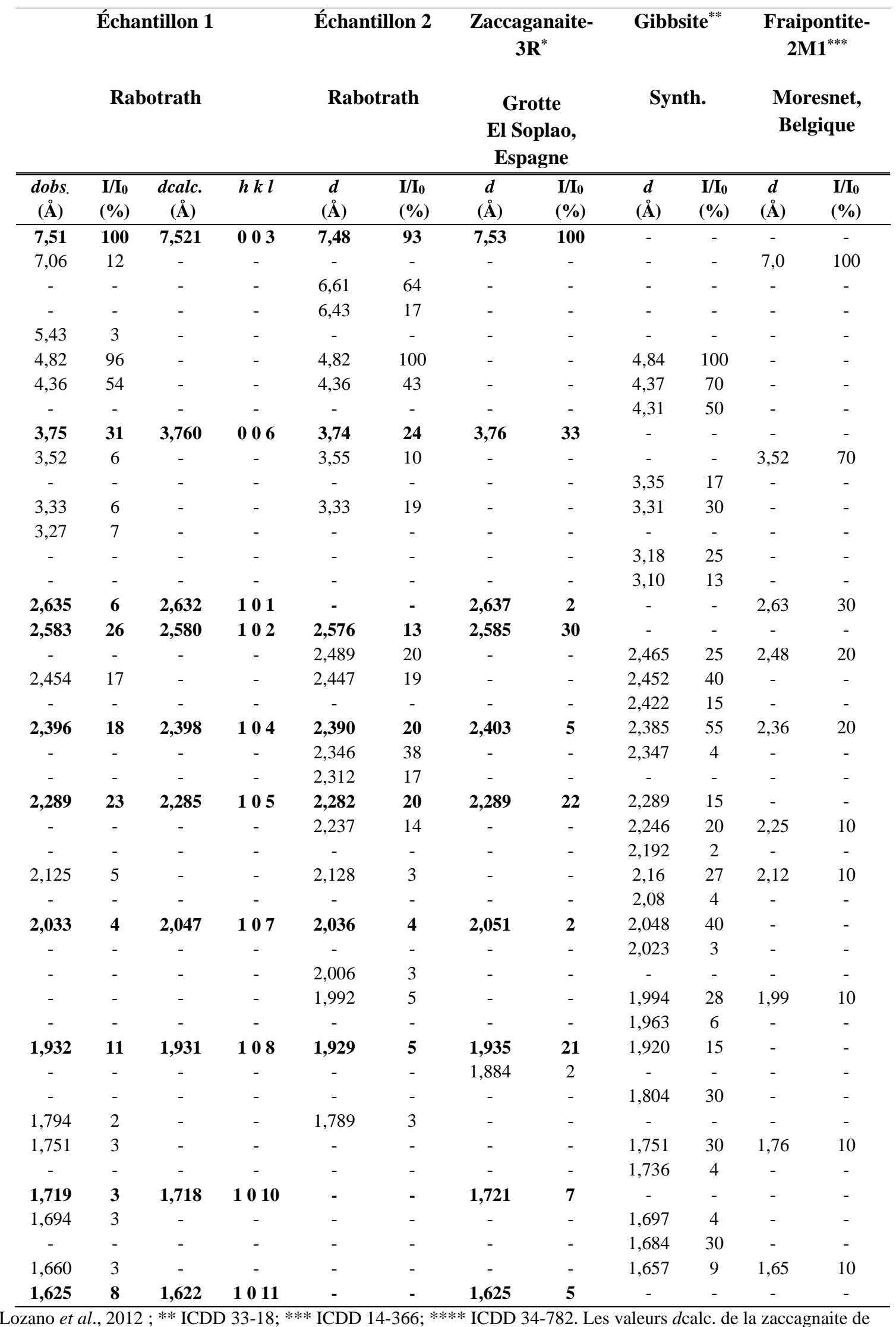

Rabotrath ont été obtenues grâce au programme LCLSQ version 8.4 (Burnham, 1991). 


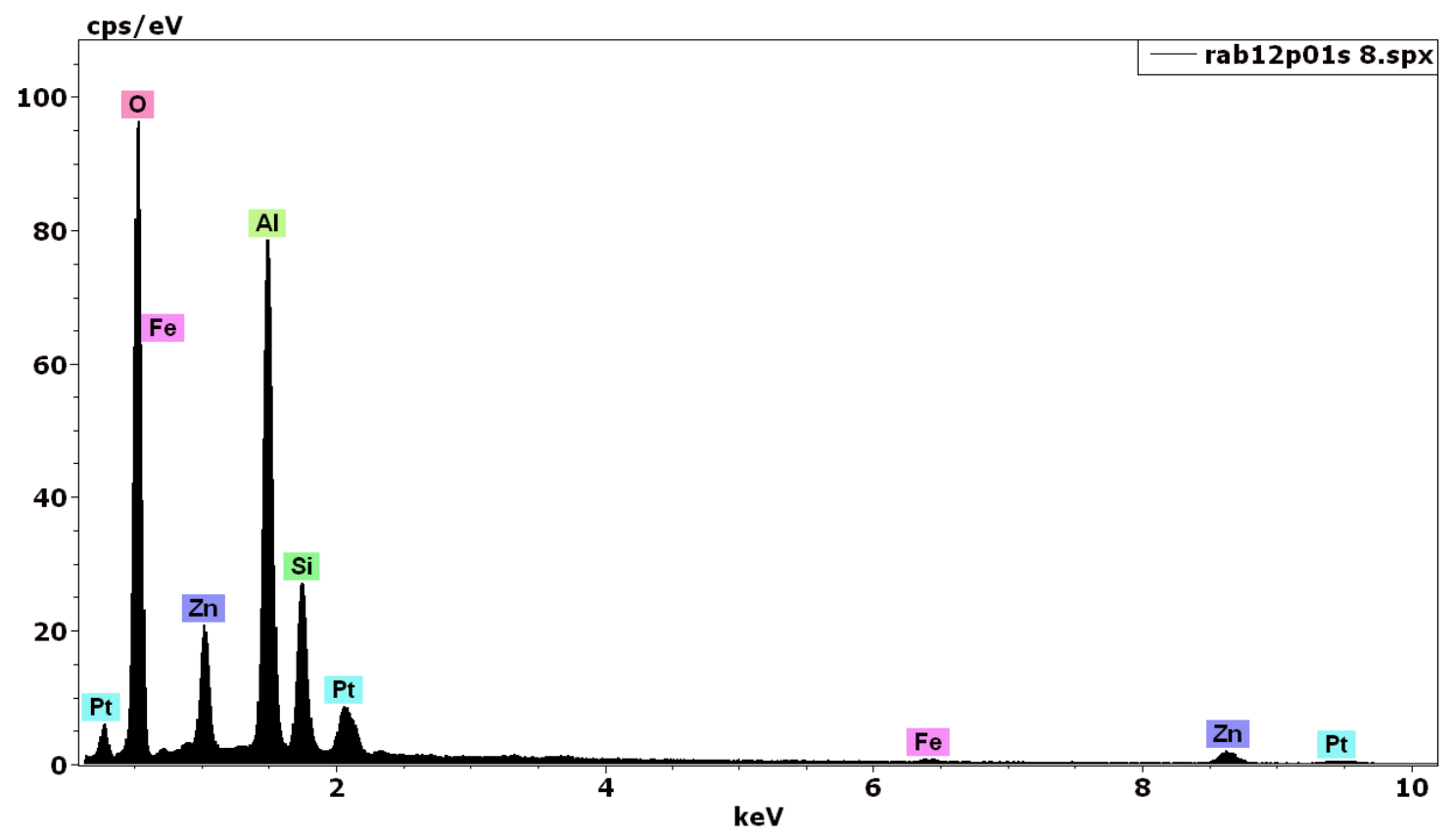

Fig. 23 : Spectre EDS de la zaccagnaite-3R de Rabotrath. Les pics du platine sont liés à la métallisation de l'échantillon.

Les 10 raies caractéristiques de la zaccagnaite-3R, observées sur le diffractogramme de poudres de l'échantillon 1 (Tableau 2), ont permis de calculer les paramètres de maille de ce minéral, grâce au programme LCLSQ version 8.4 (Burnham, 1991). Ces paramètres, $a=3.060(6)$ et $c=22.56(6) \AA$, sont en bon accord avec ceux de la zaccagnaite-3R décrite par Lozano et al. (2012) dans la grotte El Soplao, Espagne. Le gisement de Rabotrath constitue ainsi la seconde occurrence mondiale pour le polytype rhomboédrique de la zaccagnaite.

\section{Conclusion}

Le recensement des espèces minérales de Rabotrath (Tableau 3) a été favorisé par l'enlèvement d'une petite dizaine de centimètres d'épaisseur de sol, afin de favoriser l'implantation des métallophytes du zinc. Une fois le sol à nouveau colonisé par la végétation, il ne sera plus possible d'y réaliser des prélèvements.

Le petit gisement de Rabotrath, assez anecdotique d'un point de vue industriel, montre une minéralogie typique des gisements calaminaires où, à côté des sulfures minoritaires, s'observent de nombreuses espèces secondaires du zinc et du plomb. Mais, outre ces espèces assez classiques, on observe également quelques espèces moins fréquentes voire même assez rares, telles que la zaccagnaite-3R, la parascholzite, et un membre de la série goyaziteplumbogummite, qui sont trois espèces nouvelles pour la Belgique. Rabotrath est également la seconde occurrence belge pour deux autres espèces : la chalcophanite et la plumbojarosite. La présence de cérium dans la goyazite-plumbogummite laisse à penser que la présence d'espèces minérales riches en terres rares ne serait pas impossible. 
Tableau 3 : Formules chimiques idéales des espèces minérales de Rabotrath.

\begin{tabular}{|c|c|}
\hline minéral & formule chimique idéale \\
\hline Allophane & 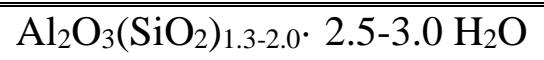 \\
\hline Anglésite & $\mathrm{PbSO}_{4}$ \\
\hline Aragonite & $\mathrm{CaCO}_{3}$ \\
\hline Calcite & $\mathrm{CaCO}_{3}$ \\
\hline Cérusite & $\mathrm{PbCO}_{3}$ \\
\hline Chalcophanite & $\mathrm{ZnMn}_{3}{ }_{3} \mathrm{O}_{7} \cdot 3 \mathrm{H}_{2} \mathrm{O}$ \\
\hline Dundasite & $\mathrm{PbAl}_{2}\left(\mathrm{CO}_{3}\right)_{2}(\mathrm{OH})_{4} \cdot \mathrm{H}_{2} \mathrm{O}$ \\
\hline Fluorapatite & $\mathrm{Ca}_{5}\left(\mathrm{PO}_{4}\right)_{3}(\mathrm{~F}, \mathrm{OH}, \mathrm{Cl})$ \\
\hline Galène & $\mathrm{PbS}$ \\
\hline Gibbsite & $\mathrm{Al}(\mathrm{OH})_{3}$ \\
\hline Goethite & $\mathrm{FeO}(\mathrm{OH})$ \\
\hline Goyazite-Plumbogummite & $(\mathrm{Sr}, \mathrm{Pb}) \mathrm{Al}_{3}\left(\mathrm{PO}_{4}\right)\left(\mathrm{PO}_{3} \mathrm{OH}\right)(\mathrm{OH})_{6}$ \\
\hline Gypse & $\mathrm{CaSO}_{4} \cdot 2 \mathrm{H}_{2} \mathrm{O}$ \\
\hline Hawleyite & $\mathrm{CdS}$ \\
\hline Hématite & $\mathrm{Fe}_{2} \mathrm{O}_{3}$ \\
\hline Marcasite & $\mathrm{FeS}_{2}$ \\
\hline Parascholzite & $\mathrm{CaZn}_{2}\left(\mathrm{PO}_{4}\right)_{2} \cdot 2 \mathrm{H}_{2} \mathrm{O}$ \\
\hline Plumbojarosite & $\mathrm{Pb}_{0,5} \mathrm{Fe}_{3}\left(\mathrm{SO}_{4}\right)_{2}(\mathrm{OH})_{6}$ \\
\hline Pyromorphite & $\mathrm{Pb}_{5}\left(\mathrm{PO}_{4}\right)_{3} \mathrm{Cl}$ \\
\hline Quartz & $\mathrm{SiO}_{2}$ \\
\hline Sidérite & $\mathrm{FeCO}_{3}$ \\
\hline Smithsonite & $\mathrm{ZnCO}_{3}$ \\
\hline Soufre & $\mathrm{S}$ \\
\hline Sphalérite & $\mathrm{ZnS}$ \\
\hline Zaccagnaite-3R & $\mathrm{Zn}_{4} \mathrm{Al}_{2}(\mathrm{OH})_{12} \mathrm{CO}_{3 .} 3 \mathrm{H}_{2} \mathrm{O}$ \\
\hline
\end{tabular}

Il est intéressant de constater aussi la présence de l'allophane, un minéral rencontré dans d'autres gisements belges comme La Rochette et le Rocheux. Ce minéral est fragile et l'érosion le fait disparaître rapidement, ce qui explique le peu d'échantillons récoltés en surface. L'allophane se forme par action météorique, en particulier dans des environnements de cryptokarsts comme à Beez. La zaccagnaite-3R de Rabotrath, associée à la gibbsite et à l'allophane, se serait-elle formée elle aussi en milieu karstique comme dans la grotte El Soplao?

En conclusion, la minéralogie de ce vieux district minier est sans doute bien plus variée et riche que généralement considéré ; des études similaires sur d'autres gisements pourraient être fort instructives à cet égard. 


\section{$\underline{\text { Remerciements : }}$}

Nous souhaitons remercier Fabrice Dal Bo et Maxime Baijot pour l'aide importante apportée lors de l'utilisation des diffractomètres au laboratoire, Francis Polrot qui a montré le site il y a une quinzaine d'années, Madame Sylvie Fabeck du musée de la Gueule à La Calamine qui a apporté des renseignements concernant la lithographie de Maugendre, ainsi que Charles Grootenclaes pour les renseignements concernant Lontzen.

\section{Références}

Blondieau, M. et Hatert, F., 2009. Minéralogie de l'ancienne mine de plomb de La Roche-enArdenne (Belgique). Le Règne Minéral, 88, 5-20.

Blondieau, M. \& Polrot, F., 2011. Les travaux miniers de Schimper, siège sud de la mine du Bleyberg (Plombières, Belgique) : plomb, zinc mais aussi argent. Geological Survey of Belgium, Professional Paper, 310, 57 p.

Blondieau, M., Hatert, F. \& Defoy, M., 2012. Minéralogie de la carrière de Beez, Province de Namur, Belgique. Le Règne Minéral, 104, 5-26.

Burnham, C.W., 1991. LCLSQ version 8.4, least-squares refinement of crystallographic lattice parameters. Dept Earth Planetary Sciences, Harvard University, $24 \mathrm{p}$

Dejonghe, L., Ladeuze, F. \& Jans, D., 1993. Atlas des gisements plombo-zincifères du synclinorium de Verviers (est de la Belgique). Mémoires pour servir à l'explication des Cartes Géologiques et Minières de la Belgique, 33, 483 p.

Dejonghe, L., 1998. Zinc-lead deposits of Belgium. Ore Geology Reviews, 12, 329-354.

Hatert, F., Deliens, M., Fransolet, A.-M. \& Van Der Meersche, E., 2002. Les minéraux de Belgique, deuxième édition, $304 \mathrm{p}$.

Kato, T., 1987. Further refinement of the goyazite structure. Mineralogical Journal, 13, 390396.

Kolitsch, U., Tiekink, E.R.T., Slade, P.G., Taylor, M.R. \& Pring, A., 1999. Hinsdalite and plumbogummite, their atomic arrangements and disordered lead sites. European Journal of Mineralogy, 11, 513-520.

Lozano, R.-P, Rossi, C., La Iglesia, A. \& Matesanz, E., 2012. Zaccagnaite-3R, a new Zn-Al hydrotalcite polytype from El Soplao cave (Cantabria, Spain). American Mineralogist, 97, 513-523. 